\title{
VLA radio continuum observations of a new sample of high redshift radio galaxies
}

\author{
L. Pentericci ${ }^{1,2}$, W. Van Reeven ${ }^{2}$, C.L. Carilli ${ }^{3}$, H.J.A. Röttgering ${ }^{2}$, and G.K. Miley ${ }^{2}$ \\ 1 Max Planck Institute für Astronomie, Königstuhl 17, 69117 Heidelberg, Germany \\ 2 Leiden Observatory, P.O. Box 9513, NL-2300 RA Leiden, The Netherlands \\ 3 NRAO, P.O. Box 0, Socorro, NM 87801, U.S.A.
}

Received March 8; accepted April 26, 2000

\begin{abstract}
We present new deep multi-frequency radiopolarimetric images of a sample of high redshift radio galaxies (HzRGs), having redshift between 1.7 and 4.1 . The radio data at 4.7 and $8.2 \mathrm{GHz}$ were taken with the Very Large Array in the A configuration and provide a highest angular resolution of $0.2^{\prime \prime}$. Maps of total intensity, radio spectral index, radio polarization and internal magnetic field are presented for each source.
\end{abstract}

The morphology of most objects is that of standard FRII double radio sources, but several contain multiple hot-spots in one or both lobes. Compared to similar samples of HzRGs previously imaged, there is a higher fraction $(29 \%)$ of compact steep spectrum sources (i.e. sources with a projected linear size less than $20 \mathrm{kpc}$ ). Radio cores are identified in about half of the sample and tend to have relatively steep spectra $(\alpha \leq-1)$.

Polarization is detected in all but 4 sources, with typical polarization at $8.2 \mathrm{GHz}$ of around $10-20 \%$. The Faraday rotation can be measured in most of the radio galaxies: the observed rotation measure $(R M)$ of 8 radio sources exceeds $100 \mathrm{rad} \mathrm{m}^{-2}$ in at least one of the lobes, with large gradients between the two lobes. We find no dependence of Faraday rotation with other properties of the radio sources. If the origin of the Faraday rotation is local to the sources, as we believe, then the intrinsic $R M$ is more than a $1000 \mathrm{rad} \mathrm{m}^{-2}$. Because low redshift radio galaxies residing at the center of clusters usually show extreme $R M \mathrm{~s}$, we suggest that the high- $z$ large $R M$ sources also lie in very dense environments.

Finally, we find that the fraction of powerful radio galaxies with extreme Faraday rotation increases with redshift, as would be expected if their average environment tends to become denser with decreasing cosmic epoch. However this result has to be taken with caution, given the limitations of our analysis.

Send offprint requests to: L. Pentericci, e-mail: laura@mpia-hd.mpg.de
Key words: galaxies: active; nuclei — radio continuum: galaxies

\section{Introduction}

High redshift radio galaxies play an important role in the study of the early universe: thanks to their extreme luminosity at different wavelengths it has been possible to use them as cosmological probes already for several decades. Currently there are more than 150 radio galaxies known with redshift greater than 2 , and recently a powerful radio source at a redshift of 5.19 has been discovered by van Breugel et al. (1999), becoming the most distant known AGN.

High redshift radio galaxies (HzRGs) comprise a different population to high redshift radio-quiet galaxies, e.g. Ly-dropouts: there is evidence that they are older and more massive, and will evolve into brightest cluster galaxies rather than $L_{*}$ ellipticals (Best et al. 1997; van Breugel et al. 1998).

In the past few years a number of studies have concentrated on the properties of HzRG host galaxies, at visual and near infrared wavelengths (e.g. Pentericci et al. 1999; van Breugel et al. 1998; Best et al. 1997; Eales et al. 1997) with the main goal of studying the morphological evolution of these host galaxies, understanding the nature of the radio-optical alignment effect and discerning the various components (stellar light, scattered light, etc.) that contribute to the optical and infrared continuum emission.

However, one of the potentially most important results from recent studies on powerful radio galaxies came from radio observations and with the discovery that a significant fraction $(\sim 20 \%)$ of HzRGs have extremely large Faraday rotation, of the order of several thousands rad $\mathrm{m}^{-2}$ (Carilli et al. 1997; Athreya et al. 1998), similar to low redshift powerful radio galaxies residing at the center 
of X-ray clusters with extreme cooling flows (Taylor et al. 1994). This makes HzRGs potential excellent targets for finding and studying high redshift (proto) clusters.

Therefore the main purpose of these new high resolution radio polarimetric imaging observations was to enlarge the number of known HzRGs with high intrinsic Faraday rotation, and to provide cluster targets for future observations with facilities such as the new X-ray telescopes, Chandra and XMM.

High resolution radio imaging is important not only for finding high Faraday rotation radio galaxies, but also for a number of other important issues, such as the identification of the location of the active nucleus, the study of the correlation between the optical morphology and the radio jets, or the line emission gas and the radio jets, and the study of the evolution of radio size structure.

Throughout the paper we adopt a cosmology with $H_{0}=50 \mathrm{~km} \mathrm{~s}^{-1} \mathrm{Mpc}^{-1}$ and $q_{0}=0.5$.

\section{Sample selection}

A large fraction of presently known HzRGs have been found by selecting ultra steep spectrum (USS) radio sources. Various groups have been involved in the search using this technique (Röttgering et al. 1994; Chambers et al. 1996; Blundell et al. 1998). At present there are about 150 radio galaxies know with $z>2$ (de Breuck et al. in preparation) of which 25 have $z>3,5$ greater than 4 and 1 has a redshift greater than 5 . About $60 \%$ of all HzRGs have been found with the USS technique and this percentage increases with redshift (e.g. de Breuck et al. 1998).

The observations presented in this paper are an extension of the sample observed by Carilli et al. (1997). We selected the present sample from the $\sim 110$ radio galaxies that were known at the time (1997). Of these 110 about 50 (most of the optically bright ones) had already been observed at high resolution (VLA in A configuration) at several frequencies (mostly by Carilli et al. 1997; few others by Carilli et al. 1994 and Athreya et al. 1997). From the remaining list we selected all the radio galaxies having total (estimated) flux density at $4.5 \mathrm{GHz}$ greater than 25 $\mu \mathrm{Jy}$, which allowed a reasonable detection of the polarized flux, even in sources with only $\sim 0.5 \%$ percentage polarization. The final selection to reduce the sample to 27 objects was done randomly. Three sources at $z<2,0152-209$, 1017-220 and 2224-273 (respectively at $z=1.89,1.77$ and 1.68) from the MRC catalogue (McCarthy et al. 1996), were added, since they are part of a sample of high redshift radio galaxies observed with HST/NICMOS (Pentericci 1999) and the VLA was needed to match the resolution of the near infrared images. Compared to the previous sample, the galaxies observed in this sample have, on average, slightly lower optical magnitudes.
In Table 1 we report the radio and optical properties of our sample: for each radio galaxy we list the radio catalogue from which it was originally taken, the redshift (usually determined from the Ly $\alpha$ emission line), and the optical magnitude of the host galaxies. In a number of cases no optical magnitude was available, either because the sources were identified by observations in a different band (e.g. $K$ band) or because they have not been measured. We also report the total flux densities at 1.4, 4.5 and $8.2 \mathrm{GHz}$ (the first value is taken from published radio catalogues listed in the table, the others are determined from the present observations), and the total extent (in arcseconds) measured from the $8.2 \mathrm{GHz}$ images for all radio sources. In the last column we list the references for the optical identification and redshift determination.

\section{Observations and data reduction}

We used the VLA in its A (27 km) configuration on 1998 March 23 and 24 to make the observations. All sources were observed at two frequencies in the $5 \mathrm{GHz}$ band of the VLA (4535 and $4885 \mathrm{MHz}$ ) and at two frequencies in the $8 \mathrm{GHz}$ band $(8085$ and $8335 \mathrm{MHz})$. Bandwidths were $50 \mathrm{MHz}$ for all frequencies. Each source was observed for 15 minutes at $8 \mathrm{GHz}$ and 8 minutes at $5 \mathrm{GHz}$.

An important limitation of using the VLA in the A configuration is the short spacing limit. This implies a maximum size on which we have information of about $10^{\prime \prime}$ at $5 \mathrm{GHz}$ and $6^{\prime \prime}$ at $8 \mathrm{GHz}$. Although this is important for the large angular scale sources, it will have a negligible effect on bright, small components such as the hot spots and cores. The data were gain-calibrated using 3C 286 . We used multiple scans of the calibrator $1745+173$ to determine the on-axis antenna polarization response. Two scans of $3 \mathrm{C} 286$ separated in time by 7 hours were used to measure the absolute linear polarization position angles.

We used the Astronomical Image Processing System (AIPS) to process the data. After calibration the data were edited and self-calibrated using standard procedures to improve image dynamic range. The first few self-calibration iterations involved phase self-calibration using a model derived from the same data. Natural weighting of the gridded visibilities was employed. The AIPS task IMAGR, in which the CLEAN algorithm is implemented, was used to deconvolve the images. The FWHM of the Gaussian restoring beams are shown in the bottom-left corners of Figs. 6-32. We synthesized images of the three Stokes parameters, $I, Q$ and $U$, and all images were CLEANed down to the noise-levels. The achieved noise is $25 \mu \mathrm{Jy} /$ beam at $8 \mathrm{GHz}$ and 50 $\mu \mathrm{Jy} /$ beam at $5 \mathrm{GHz}$. The resolution of the observations is $0.23^{\prime \prime}$ for the $8.2 \mathrm{GHz}$ maps, and $0.43^{\prime \prime}$ for the $4.7 \mathrm{GHz}$ maps.

Total intensity maps were created using the combined data from the two frequencies per band and the mean frequencies in each band. In order to make spectral index 
Table 1. Source parameters

\begin{tabular}{|c|c|c|c|c|c|c|c|c|}
\hline $\begin{array}{c}\text { Source } \\
\text { (B1950) } \\
(1)\end{array}$ & $\begin{array}{c}z \\
(2)\end{array}$ & $\begin{array}{c}\text { Catalog } \\
(3)\end{array}$ & $\begin{array}{c}R \\
(\mathrm{mag}) \\
(4)\end{array}$ & $\begin{array}{c}\text { Size } \\
(\operatorname{arcsec}) \\
(5)\end{array}$ & $\begin{array}{c}F_{1.4} \\
(\mathrm{mJy}) \\
(6)\end{array}$ & $\begin{array}{c}F_{4.7} \\
(\mathrm{mJy}) \\
(7)\end{array}$ & $\begin{array}{c}F_{8.2} \\
(\mathrm{mJy}) \\
(8)\end{array}$ & $\begin{array}{l}\text { Ref. } \\
(9)\end{array}$ \\
\hline $0011-023$ & 2.080 & PKS & 23.5 & $<0.2$ & $347^{a}$ & 161 & 95 & 1 \\
\hline $0152-209$ & 1.89 & MRC & 21.9 & 2.2 & $453^{b}$ & 109 & 47 & 2 \\
\hline $0930+389$ & 2.395 & $6 \mathrm{C}$ & - & 4.2 & $215^{c}$ & 73 & 33 & 3 \\
\hline $1017-220$ & 1.77 & MRC & 21.6 & $<0.2$ & $583^{d}$ & 257 & 148 & 2 \\
\hline $\mathrm{J} 1019+053$ & 2.765 & MG & 23.7 & 2.2 & $454^{a}$ & 120 & 59 & 4 \\
\hline $1031+34$ & 2.1 & $6 \mathrm{C}$ & 21.4 & 41.2 & $478^{a}$ & 128 & 56 & 3 \\
\hline $1039+681$ & 2.530 & $8 \mathrm{C}$ & - & 16.6 & $268^{a}$ & 61 & 25 & 5 \\
\hline $1056+39$ & 2.171 & B2 & 23.6 & 14.2 & $264^{a}$ & 71 & 34 & 3 \\
\hline $1132+37$ & 2.88 & B2 & - & $<0.2$ & $637^{c}$ & 227 & 127 & 3 \\
\hline $1134+369$ & 2.120 & $6 \mathrm{C}$ & - & 14.0 & $235^{a}$ & 61 & 26 & 3 \\
\hline $1202+527$ & 2.73 & $\mathrm{TX}$ & - & 5.3 & $441^{a}$ & 188 & 162 & 6 \\
\hline $1204+401$ & 2.066 & B3 & 23.0 & 2.5 & $237^{c}$ & 60 & 28 & 7 \\
\hline J1338-19 & 4.11 & $\mathrm{TN}$ & 22.0 & 5.2 & - & 23 & 9 & 8 \\
\hline $1339+35$ & 2.772 & $\mathrm{FW}$ & - & 13.0 & $124^{c}$ & 21 & 9 & 9 \\
\hline $1357+007$ & 2.671 & PKS & 23.7 & 2.7 & $296^{a}$ & 59 & 32 & 10 \\
\hline $1425-148$ & 2.355 & PKS & 22.0 & 11.6 & $413^{d}$ & 120 & 53 & 9 \\
\hline $1558-003$ & 2.520 & $\mathrm{TX}$ & 23.4 & 9.2 & $375^{a}$ & 91 & 38 & 10 \\
\hline $1647+100$ & 2.509 & $\mathrm{TX}$ & 23.5 & 23.4 & $298^{a}$ & 44 & 18 & 9 \\
\hline $\mathrm{J} 1747+182$ & 2.281 & $\mathrm{MG}$ & 23.0 & 7.3 & $1095^{a}$ & 329 & 166 & 11 \\
\hline $1908+722$ & 3.537 & $6 \mathrm{C}$ & 21.4 & 15.4 & $259^{a}$ & 49 & 16 & 12 \\
\hline $2034+027$ & 2.129 & $\mathrm{TX}$ & 24 & 3.9 & $243^{a}$ & 61 & 26 & 2 \\
\hline $2048-272$ & 2.06 & MRC & $>24$ & 6.7 & $457^{e}$ & 90 & 35 & 2 \\
\hline $2052-253$ & 2.630 & $\mathrm{MRC}$ & 23.8 & 20.0 & $219^{e}$ & 49 & 22 & 2 \\
\hline $2104-242$ & 2.49 & MRC & 22.7 & 23.7 & $297^{e}$ & 58 & 21 & 2 \\
\hline $2211-251$ & 2.508 & MRC & 23.4 & 3.5 & $836^{d}$ & 227 & 112 & 2 \\
\hline $2224-273$ & 1.68 & MRC & 22.5 & $<0.2$ & $233^{e}$ & 48 & 19 & 2 \\
\hline $2319+223$ & 2.554 & $\mathrm{TX}$ & - & 8.9 & $284^{a}$ & 44 & 17 & 9 \\
\hline
\end{tabular}

(1) Most common name in B1950 notation (except when there is a $\mathrm{J}$ ); (2) redshift; (3) Radio catalogue: MRC Molonglo reference catalogue; TX Texas; PKS Parkes; MG MIT-Greenbank; 6C/8C Cambridge; B2 Second Bologna; (4) $R$-band magnitude of the host galaxies (when available); (5) Total angular extent of the radio source; (6) Total flux density at 1.4 GHz: ${ }^{a}$ Green Bank $1.4 \mathrm{GHz}$, White and Becker 1992; ${ }^{b}$ NVSS, Condon et al. 1998; ${ }^{c}$ FIRST, Becker et al. 1995; ${ }^{d}$ extrapolated from the $408 \mathrm{MHz}$ flux of the PKSCAT90, Wright and Otrupcek (Eds) 1990; ${ }^{e}$ extrapolated from the $408 \mathrm{MHz}$ flux of the MRC Catalogue, Lange et al. 1981; (7) Total flux density at $4.7 \mathrm{GHz}$; (8) Total flux density at $8.2 \mathrm{GHz}$; (9) Reference for redshift: 1 . Dunlop et al. 1989; 2. McCarthy et al. 1996; 3. Eales and Rawlings 1996; 4. Dey et al. 1995; 5. Lacy, Ph.D. Thesis; 6. Owen et al. $1995 ; 7$. Thompson et al. 1994; 8. Rawlings and Lacy 1996; 9. De Breuck et al. in preparation; 10. Röttgering et al. 1997; 11. Eales and Rawlings 1993; 12. Dey et al. 1998.

maps we convolved the $8 \mathrm{GHz}$ image with the Gaussian restoring beam of the $5 \mathrm{GHz}$ image. Since the spectrum of the sources can be approximated by a power law, the spectral index $\alpha$ is defined as as $I_{\nu} \propto \nu^{\alpha}$, where $I_{\nu}$ is the surface brightness at frequency $\nu$. We calculated two-point spectral index values only for pixels with surface brightness exceeding $3.5 \sigma$ (where $\sigma$ is the measured off-source rms on an image) at both frequencies.

We used position angles for the polarized intensity for three frequencies $(4535,4885$ and $8200 \mathrm{MHz})$ to derive rotation measures. The rotation measures were derived using the AIPS task $R M$. For the hot-spots, rotation measures were derived for the position of peak intensity.

\section{Images and observed parameters}

For each source four images are presented (Figs. 6-32). They represent the total intensity at $4.7 \mathrm{GHz}$ (upper left), the total intensity at $8.2 \mathrm{GHz}$ (upper right), the spectral index between $4.7 \mathrm{GHz}$ and $8.2 \mathrm{GHz}$ (lower right) and the polarized intensity at $4.7 \mathrm{GHz}$ (lower left) with overlayed vectors indicating the position angles of the magnetic field. The two total intensity maps are at full resolution, the spectral index map and polarized intensity map are at the resolution of the $4.7 \mathrm{GHz}$ image.

For some of the sources we had to rotate the image over 90 degrees in order to best display the source structure. The four images are placed in the same way as with the other sources. 
Table 2. Core parameters

\begin{tabular}{|c|c|c|c|c|}
\hline \multirow{3}{*}{$\begin{array}{l}\text { Source } \\
\text { (1) }\end{array}$} & Core Position(J2000) & \multirow{3}{*}{$\begin{array}{c}F_{8.2} \\
\left(\text { mJy beam }^{-1}\right) \\
(3)\end{array}$} & \multirow{3}{*}{$\begin{array}{r}\alpha_{4.7}^{8.2} \\
(4) \\
\end{array}$} & \multirow{3}{*}{$\begin{array}{c}C F_{20} \\
(\%) \\
(5) \\
\end{array}$} \\
\hline & Dec & & & \\
\hline & $(2)$ & & & \\
\hline $0011-023$ & $001425.54-020555.1$ & 88.4 & -0.9 & 100.0 \\
\hline $0152-209$ & $\ldots$ & $\ldots$ & $\ldots$ & $\ldots$ \\
\hline $0930+389$ & $093306.94+384150.8$ & 0.29 & -0.8 & 0.8 \\
\hline $1017-220$ & $101949.02-221959.8$ & 144.7 & -1.0 & 100.0 \\
\hline $1019+053 \mathrm{~J}$ & $101933.42+053434.8$ & $\mathrm{X} 2.06$ & -1.0 & 4.7 \\
\hline $1031+34$ & $103434.61+334927.3$ & 0.38 & -0.3 & 0.6 \\
\hline $1039+681$ & $\ldots$ & $\ldots$ & $\ldots$ & $<0.23$ \\
\hline $1056+39$ & $\ldots$ & $\ldots$ & $\ldots$ & $<0.13$ \\
\hline $1132+37$ & $113505.93+370840.8$ & 123.9 & -1.0 & 100.0 \\
\hline $1134+369$ & $\ldots$ & $\ldots$ & $\ldots$ & $<0.16$ \\
\hline $1202+527$ & $\ldots$ & $\ldots$ & $\ldots$ & $<0.04$ \\
\hline $1204+401$ & $120706.27+395439.0$ & 0.66 & -0.5 & 2.1 \\
\hline $1338-19 \mathrm{~J}$ & $133826.23-194233.6$ & 0.16 & -1.0 & 1.9 \\
\hline $1339+35$ & $\cdots$ & $\ldots$ & $\ldots$ & $<0.44$ \\
\hline $1357+007$ & $140021.26+003020.7$ & 10.3 & -0.4 & 27.0 \\
\hline $1425-148$ & $142841.72-150228.4$ & 0.35 & -0.7 & 0.5 \\
\hline $1558-003$ & $160117.36-002846.3$ & 0.97 & -0.3 & 1.6 \\
\hline $1647+100$ & $\ldots$ & $\ldots$ & $\ldots$ & $<0.17$ \\
\hline $1747+182 \mathrm{~J}$ & $\ldots$ & $\ldots$ & $\ldots$ & $<0.28$ \\
\hline $1908+722$ & $190909.74+721515.3$ & 1.2 & -1.3 & 4.8 \\
\hline $2034+027$ & $203634.78+025654.4$ & 1.7 & -1.2 & 6.1 \\
\hline $2048-272$ & $\ldots$ & $\ldots$ & $\ldots$ & $<0.18$ \\
\hline $2052-253$ & $\ldots$ & $\ldots$ & $\ldots$ & $<2.3$ \\
\hline $2104-242$ & $210658.27-240509.1$ & 0.19 & -1.6 & 0.7 \\
\hline $2211-251$ & $\ldots$ & $\ldots$ & $\ldots$ & $<0.04$ \\
\hline $2224-273$ & $222743.27-270501.7$ & 17.8 & -1.6 & 100.0 \\
\hline $2319+223$ & $\ldots$ & $\ldots$ & $\ldots$ & $<0.25$ \\
\hline
\end{tabular}

(1) Source name, (2) position of the radio core in J2000 coordinates; (3) core surface brightness in mJy beam ${ }^{-1}$, measured on the $8.2 \mathrm{GHz}$ map, convolved to the resolution of the $4.7 \mathrm{GHz}$ image, unless preceeded by an "X"; (4) core spectral index between $4.5 \mathrm{GHz}$ and $8.2 \mathrm{GHz}$; (5) percentage core fractions calculated at a rest frame frequency of $20 \mathrm{GHz}$, with upper limits for the undetected cores.

In the intensity maps, the contour levels vary with $2^{1 / 2}$, which implies a change in surface brightness of a factor 2 every 2 contours including the negative ones. The first non-negative contour is indicated in the caption of each image and is set to $3 \sigma$, with $\sigma$ the measured off-source rms. The peak surface brightness in each image is also given in the caption.

The contour levels in the spectral index maps are -3 , $-2.8,-2.6,-2.4,-2.2,-2,-1.8,-1.6,-1.4,-1.2,-1$, $-0.8,-0.6,-0.4,-0.2$ and 0 . The grey scale in the spectral index images also ranges from -3 to 0 .

In Tables 2 and 3 some of the observed properties of the sources are listed. All source identifiers are for the B1950.0 coordinate system except those marked with J. Table 2 lists the properties of the radio cores, where identified. In Col. (2) we list core positions (J2000 coordinates), in Col. (3) the flux densities of the core at $8.2 \mathrm{GHz}$, in Col. (4) the spectral index between $4.7 \mathrm{GHz}$ and $8.2 \mathrm{GHz}$. Column (5) lists the core fraction at a rest frame frequency of $20 \mathrm{GHz}$.
Table 3 lists properties of the brightest southern and northern hot spots. Columns (2)-(5) list the properties of the southern hot spot, while Cols. (6)-(10) list the properties of the northern hot spot. They are the peak surface brightness, the spectral index between 4.7 and $8.2 \mathrm{GHz}$, the hot spot fractional polarization at 4.7 and $8.2 \mathrm{GHz}$ and the observed rotation measure at the hot spot position respectively. The $8.2 \mathrm{GHz}$ fractional polarization was obtained in images that were convolved to the $4.7 \mathrm{GHz}$ resolution.

\section{Discussion}

\subsection{Morphology and core identification}

All the resolved sources have a FRII double morphology, with hot-spots at the extremity of the radio source. A few sources, such as 1558-003, have multiple hot-spots connecting the core to the outermost components, typically on only one side. There are 8 small sources that can be 
Table 3. Hot-Spots parameters

\begin{tabular}{|c|c|c|c|c|c|c|c|c|c|c|}
\hline \multirow[b]{2}{*}{$\begin{array}{c}\text { Source } \\
(1)\end{array}$} & \multicolumn{5}{|c|}{ "Southern Hot Spot } & \multicolumn{5}{|c|}{ Northern Hot Spot } \\
\hline & $\begin{array}{c}I_{4.5} \\
\text { mJy/beam } \\
(2)\end{array}$ & $\begin{array}{r}\alpha_{4.7}^{8.2} \\
(3)\end{array}$ & $\begin{array}{c}F P_{4.5} \\
(\%) \\
(4)\end{array}$ & $\begin{array}{c}F P_{8.2} \\
(\%) \\
(5)\end{array}$ & $\begin{array}{c}R M \\
\left(\operatorname{rad~m^{-2}}\right) \\
(6)\end{array}$ & $\begin{array}{c}I_{4.5} \\
\mathrm{mJy} / \text { beam } \\
(7)\end{array}$ & $\begin{array}{r}\alpha_{4.7}^{8.2} \\
(8)\end{array}$ & $\begin{array}{c}F P_{4.5} \\
(\%) \\
(9)\end{array}$ & $\begin{array}{c}F P_{8.2} \\
(\%) \\
(10)\end{array}$ & $\begin{array}{c}R M \\
\left(\operatorname{rad~m^{-2}}\right) \\
(11)\end{array}$ \\
\hline $0011-023$ & 149.4 & -0.9 & $\ldots$ & $\ldots$ & $\ldots$ & $\ldots$ & $\ldots$ & $\ldots$ & $\ldots$ & $\ldots$ \\
\hline $0152-209$ & 95.9 & -1.4 & $\ldots$ & 0.4 & $\ldots$ & 7.2 & -1.7 & 0.3 & 2.4 & 87 \\
\hline $0930+389$ & 30.1 & -1.3 & 1.6 & 7.4 & 267 & 28.7 & -1.3 & 4.0 & 2.9 & 29 \\
\hline $1017-220$ & 253.0 & -1.0 & $\ldots$ & $\ldots$ & $\ldots$ & $\ldots$ & $\ldots$ & $\ldots$ & $\ldots$ & $\ldots$ \\
\hline $1019+053 \mathrm{~J}$ & 48.5 & -1.1 & 0.2 & 6.0 & $\ldots$ & 51.6 & -1.2 & 1.2 & 4.5 & -36 \\
\hline $1031+34$ & 48.3 & -1.1 & 6.9 & 7.8 & 51 & 8.7 & -1.5 & 9.3 & 6.9 & 19 \\
\hline $1039+681$ & 21.6 & -1.1 & 7.9 & 15.4 & -170 & 3.5 & -1.4 & 21.1 & 34.7 & -65 \\
\hline $1056+39$ & 9.7 & -1.1 & 13.4 & 17.9 & 2 & 42.8 & -1.2 & 4.4 & 11.1 & -95 \\
\hline $1132+37$ & 218.6 & -1.0 & $\ldots$ & $\ldots$ & $\ldots$ & $\ldots$ & $\ldots$ & $\ldots$ & $\ldots$ & $\ldots$ \\
\hline $1134+369$ & 10.1 & -1.4 & 8.1 & 15.9 & 140 & 29.8 & -1.1 & 4.0 & 6.3 & 4 \\
\hline $1202+527$ & 6.0 & -1.6 & 5.8 & 12.4 & 208 & 154.4 & -0.1 & 3.0 & 0.8 & -100 \\
\hline $1204+401$ & 16.6 & -1.4 & $\ldots$ & $\ldots$ & $\ldots$ & 22.3 & -1.1 & 5.1 & 16.2 & $\ldots$ \\
\hline 1338-19J & 4.7 & -1.8 & $\ldots$ & $\ldots$ & $\ldots$ & 20.6 & -1.6 & $\ldots$ & 1.7 & $\ldots$ \\
\hline $1339+35$ & 2.9 & -2.5 & $\ldots$ & $\ldots$ & $\ldots$ & 12.4 & -2.2 & 1.9 & 3.6 & -160 \\
\hline $1357+007$ & 17.3 & -1.2 & 1.8 & 1.8 & -111 & 18.0 & -1.2 & $\ldots$ & 1.2 & $\ldots$ \\
\hline $1425-148$ & 30.3 & -1.5 & 3.1 & 3.4 & 108 & 50.2 & -1.4 & 17.4 & 19.7 & -27 \\
\hline $1558-003$ & 39.9 & -1.3 & 11.0 & 13.0 & -15 & 75.7 & -1.5 & 10.9 & 5.4 & 36 \\
\hline $1647+100$ & 8.2 & -1.3 & 1.9 & 1.7 & $\ldots$ & 24.6 & -1.4 & 5.3 & 6.3 & 12 \\
\hline $1747+182 \mathrm{~J}$ & 161.7 & -1.1 & 7.5 & 16.6 & 73 & 92.2 & -1.0 & 21.6 & 31.7 & 62 \\
\hline $1908+722$ & 16.0 & -1.7 & 7.3 & 6.1 & 34 & 7.3 & -1.8 & 13.9 & 16.8 & 91 \\
\hline $2034+027$ & 31.2 & -1.3 & 0.6 & 4.6 & -45 & 9.8 & -1.6 & 9.2 & 18.9 & $\ldots$ \\
\hline $2048-272$ & 1.4 & -3.1 & $\ldots$ & $\ldots$ & $\ldots$ & 49.7 & -1.4 & 7.7 & 12.3 & 63 \\
\hline $2052-253$ & 2.9 & -1.8 & 9.3 & 26.9 & $\ldots$ & 11.8 & -1.9 & 6.0 & 16.8 & -62 \\
\hline $2104-242$ & 2.3 & -1.9 & 6.6 & $\ldots$ & $\ldots$ & 21.8 & -1.5 & 14.9 & 16.1 & -7 \\
\hline $2211-251$ & 18.9 & -1.5 & 6.3 & $\ldots$ & 54 & 190.1 & -1.2 & 0.25 & $\ldots$ & $\ldots$ \\
\hline $2224-273$ & 45.9 & -1.6 & $\ldots$ & $\ldots$ & $\ldots$ & $\ldots$ & $\ldots$ & $\ldots$ & $\ldots$ & $\ldots$ \\
\hline $2319+223$ & 32.4 & -1.6 & 8.6 & 10.8 & 50 & 7.5 & -1.4 & 5.3 & 9.7 & 10 \\
\hline
\end{tabular}

Columns (2)-(6) refer to the southern hot-spots, and (7)-(11) to the northern one. (2) and (7) are the peak surface brightness; (3) and (8) the spectral index; (4) and (9) the fractional polarization at $4.7 \mathrm{GHz}$; (5) and (10) the fractional polarization at $8.2 \mathrm{GHz}$ : (6) and (11) the rotation measure calculated using observed frequencies with no correction for redshift. A blank entry means that there was no detection at a $3 \sigma$ level.

classified as compact steep spectrum (CSS) sources, having linear (projected) sizes smaller than about $20 \mathrm{kpc}$ (e.g. Fanti et al. 1990). Of these, 3 are unresolved (1017-220 and $1132+37$ and $2224-273$, with sizes less than $0.2^{\prime \prime}$ ), one is barely resolved (0011-023, which is sightly elongated in the $4.7 \mathrm{GHz}$ maps) and another 4 (0152-209, $1019+053,1204+401$ and $1357+007)$ appear as small FRII radio galaxies. The percentage of CSS sources is $29 \%$, significantly higher than found in similar samples observed at the same frequencies and VLA array configuration: $14 \%$ of small sources in the sample of Carilli et al. (1997), which included $38 \mathrm{HzRGs}$; $13 \%$ in the sample of Athreya et al. (1997), which included 15 HzRGs, some of which also belonged to the previous sample. The only difference between our sample and the previous ones, is the slightly lower average optical magnitude of the radio galaxies selected here, but it is unclear how this should influence the average size of the sources. Interestingly the percentage of CSS sources in this study is more similar to that found by
Lonsdale et al. (1993) in a sample of steep spectrum radio loud quasars at $z \geq 1.5$ (27\% of CSS sources).

In Table 2 we tabulate the characteristics (coordinates, flux density, spectral index, and core fraction) of the cores. We can identify the radio cores in less than half of the resolved radio sources (11 out of 24): the cores are identified as the unresolved component with the relatively flattest spectral index, which are not polarized. The percentage of identified cores is lower that the identification rate of Carilli et al. (1997) (75\%).

In the source $1202+527$ there is a component with very flat spectral index $(-0.1)$, but given that it is polarized and that its position would imply a highly asymmetric morphology, we consider it to be a hot-spot, and tabulate its characteristics in Table 3 instead of Table 2. In the highest redshift radio source of the sample, 1338-19 at $z=4.11$, the core is the faint component just below the northern hot-spot, and appears only in the high frequency map (Fig. 18b). Despite its steep spectrum $(\alpha=-1)$ and 
the high resulting asymmetry of the radio sources we are confident about its identification given its proximity to the host galaxy (de Breuck et al. 1999).

As previously mentioned, in general the radio cores are identified as the flat spectrum components: however in 5 radio galaxies the cores have very steep spectra (spectral index equal or steeper than -1 ). While it could be that in some cases the nucleus is not correctly identified or is blended with a steep spectrum component (this could be the case of $1019+681$ ), most should be true unresolved cores.

Steep core spectra have previously been found in many high redshift radio galaxies by Carilli et al. (1997) and Athreya et al. (1997). Athreya et al. suggest that the rest frame frequencies at which these cores are observed (15 to $30 \mathrm{GHz}$, depending on redshift) are higher than the turn over frequency due to synchrotron self-absorption. They suggest that the cores of radio sources exhibit synchrotron self-absorption turn over at $20 \mathrm{GHz}$ in the rest frame of the emitting plasma, and that the spectra will appear steep above that frequency. They also explain the difference between the galaxy cores and quasars cores (which exhibit steepening at a much higher frequency) with the fact that the turn over frequency appears blue-shifted in the relativistically beamed quasar core and redshifted in the galaxy cores. They predict that the size of the dominant core component should be less than 1 mas.

In Col. 5 of Table 2 we list the core factions, calculated at a rest-frame frequency of $20 \mathrm{GHz}$ : for those sources where the core is undetected we give an upper limit, assuming that the core flux is less than 3 times the rms noise of the map. Core fractions vary from less than $0.05 \%$ to few $\%$, a range that is typical for radio galaxies. The only exception is the CSS source $1357+007$ that has a core contributing for $27 \%$ of the total flux. The median core fraction is $0.6 \%$.

According to evolutionary models of radio sources (e.g. Kaiser et al. 1997), as a radio source grows older and expands, its lobe radio luminosity declines, whereas the core flux remains constant. Therefore the prediction is that larger radio sources, which on average should be older, should also have higher core fractions. To test this model, in Fig. 1a we have plotted the core fractions of the radio sources in this sample, augmented by the sample of Carilli et al. (1997), versus their radio sizes in kpc. We do not detect any increase in the average core fractions with increasing radio size: on the contrary we see the opposite effect, i.e. a slight decrease in the core fraction, from a median value of $1.5 \%$ for sources smaller than $50 \mathrm{kpc}$, to $1.2 \%$ for sources between 50 and 100 , to $0.8 \%$ for sources larger than $100 \mathrm{kpc}$, with a large scatter around these median values at any given size (note that using the median value is a better estimate, expecially when dealing with upper limits).

These trends should be interpreted with caution since the sizes of HzRGs range only from 10 to less than $500 \mathrm{kpc}$ and there are very few large sources. For example the only two sources larger than $400 \mathrm{kpc}$ have relatively large core fractions. Therefore if the effect predicted by Kaiser et al. sets in only at rather large sizes (of few hundreds of kpc), we would not observe it given our limited number of large sources.

The core fractions of HzRGs tend to be higher than those of matched luminosity 3CR galaxies (Laing et al. 1983) at redshift $z \sim 1$. Best et al. (1999) show that for these radio galaxies the median core fraction at a rest frame frequency of $16 \mathrm{GHz}$ is $0.2-0.3 \%$ and does not depend on radio sources size for a large range of sizes (from 10 to $1000 \mathrm{kpc}$. The slight difference in rest-frame frequency (we use $20 \mathrm{GHz}$ instead of $16 \mathrm{GHz}$ ) should not be important. Therefore the core fractions at $z>2$ are $\sim 4$ times larger that at $z \sim 1$. This could indicate that either at high redshift there are intrinsically stronger cores, or that the beaming factor is higher at earlier epochs. Alternatively, if the core fraction really depends on radio sources sizes as predicted by Kaiser et al. (1997), the difference between the high and low redshift samples could be due to different average sizes of the two samples considered. A full discussion of this issue is beyond the scope of this paper.

In Fig. 1b we present also a plot of core fraction versus power. Although we sample only about one order of magnitude in power, we see no significant correlation between these two quantities. This is in agreement with previous results at lower redshift (Best et al. 1999).

\subsection{Radio source distortion}

Several sources in our sample show "distorted" morphologies, with multiple hot-spots which are often not aligned (e.g. 1908+722). Following previous authors (e.g. Barthel \& Miley 1988), we measure this non-linearity of a radio source with a "bending angle", which is defined as $180^{\circ}$ minus the angle between the lines joining the core to opposite hot-spots on either side of the source. The bending angles for objects in our sample range from $0^{\circ}$ to $22^{\circ}$. Note that many sources such as $1908+722$ and $1039+681$ show multiple bends, therefore the bending angle as defined above only measures the overall bends as defined by the outer extremities.

In Fig. 2a we present the distribution of the bending angles of our present sample, with the addition of data for objects in Carilli et al. (1997), supplemented by data on a few radio sources from the literature (Carilli et al. 1994). This larger sample is homogeneous, i.e. all the galaxies have been observed at the same resolution and frequencies. The distribution of bending galaxies is basically flat from $0^{\circ}$ to $20^{\circ}$, with an average of $12.3^{\circ}$. We compare this distribution with that of the $3 \mathrm{CR}$ radio sources which are matched in luminosity and have redshifts between 0.6 and 1.6 (Fig. 2b reproduced from Athreya 1996). The difference is striking: not only is the average bending angle at 

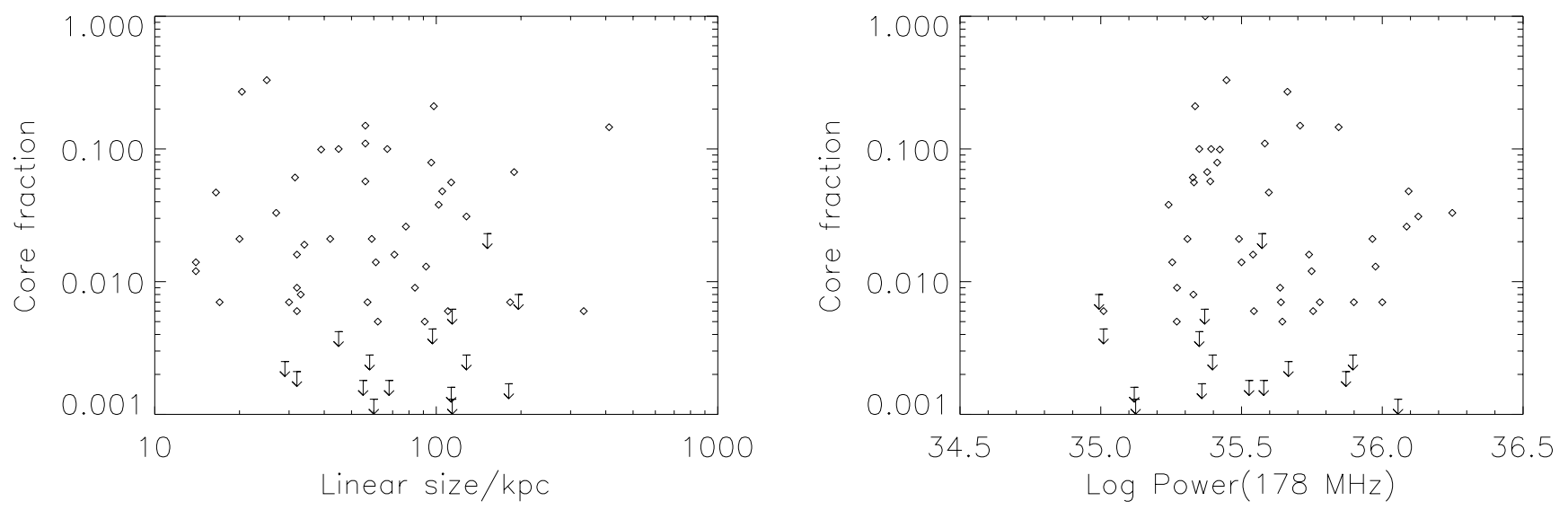

Fig. 1. Left: A log-log plot of the core fraction at rest frame frequency of $20 \mathrm{GHz}$ versus linear radio size in kpc (arrows represent upper limits). Right: The core fraction plotted versus the radio luminosity at a rest-frame frequency of $178 \mathrm{MHz}$

redshift greater than 2 more than double that at $z \sim 1$, but also the distribution is different.

Barthel \& Miley (1988) first pointed out the increasing distortion in the appearance of radio sources at high redshift, although the angles involved for quasars are larger, due to larger projection angles (e.g. Kaphai 1990). This is in agreement with the predictions of unification schemes based on orientation. The increase with redshift of asymmetries in the morphology of powerful radio galaxies was also noted by McCarthy et al. (1991) for low redshift $3 \mathrm{CR}$ radio sources, comparing two samples of $z<0.2$ and $2.5>z>0.7$ radio galaxies.

A source can be distorted due to interaction between the radio jets and the ambient medium: one example is the radio galaxy $1138-262$ (Pentericci et al. 1998), which shows clear signs of interaction between the western jet and the emission line gas, and has large velocity gradients at the location where the radio jet sharply bends. Therefore denser and clumpier environment at high redshift could also explain the increase of distortion with redshift. Indeed optical and narrow band observations have shown that also the stars and gas distribution of HzRGs appear extremely clumpy and asymmetric at high redshift, on a scale comparable or larger than that of the radio emission (e.g. Pentericci et al. 1999).

The increase of ambient density with redshift has also been invoked to explain the decrease in average source size, at a fixed radio power, observed by many groups, although there is still disagreement on the exact shape of the distance-redshift relationship (see references in Blundell et al. 1999).

\subsection{Polarization properties}

In most sources one or more components are polarized at both frequencies; typical polarization levels are on the or- der of less than $10 \%$ at $4.5 \mathrm{GHz}$ and up to $20 \%$ at $8.2 \mathrm{GHz}$. In many case there are large differences in polarization between the hot-spots, which could be due to asymmetric properties in the environment.

In Figs. 6-32c we present the polarization maps at $4.5 \mathrm{GHz}$ with superimposed vectors representing the direction and strength of the magnetic field (corrected for Faraday rotation). The electric field is oriented perpendicular to these vectors.

In about half the sources the hot-spots magnetic fields are oriented perpendicular to the jet direction, a common characteristic of the hot-spots of powerful radio sources (e.g. Muxlow \& Garrington 1991). However there are several cases, such as $1357+007$ where the magnetic field vectors are parallel to the jet direction, and in many sources both parallel and perpendicular fields are present (e.g. 2211-251).

A possible reason is that these components are not true outer hot-spot but are associated with jets or oblique shocks. The magnetic field is parallel in the jets, and perpendicular to the jet axis in the hot-spots, while in the radio bridges it wraps around the the edges around the hot-spots, hence being parallel to the radio axis (Saikia \& Salter 1988). Therefore if the knots we observe are not real hot-spots but consist of different unresolved structures, there can be intermediate direction or even parallel $B$ field.

The strength of the magnetic field in each hot-spot can be calculated by making the standard minimum energy conditions (Miley 1980): the resulting magnetic fields range from 160 to $700 \mu \mathrm{G}$.

\subsection{Faraday rotation}

In Table 3 we list the observed values of Faraday rotation for those regions of the radio galaxies that produce enough 

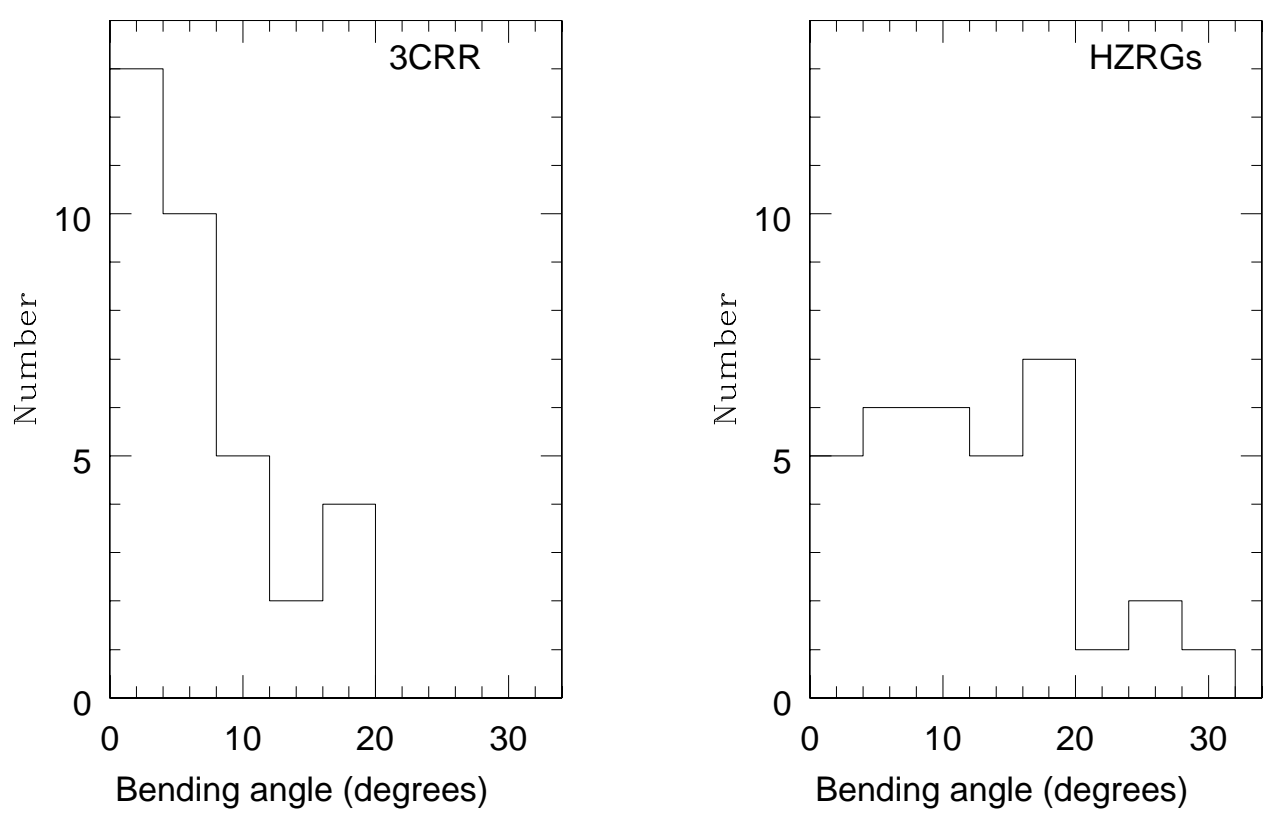

Fig. 2. Right: the distribution of the bending angles for the sample of HzRGs that includes all the sources presented in this paper plus those from Carilli et al. (1997). Left, the analogous distribution for the 3CR sources (figure from Athreya 1997)

polarized signal to allow a determination of the angle of polarization (see Sect. 2). In Fig. 3 we show several plots of the polarization position angles (in radians) versus wavelength squared for the components of three HzRGs. The lines represent the best linear fit to the data points, given by the AIPS task $R M$.

If the Faraday screen is located at a redshift $z_{\mathrm{F}}$, then the intrinsic value of $R M_{\text {intr }}$ is related to the observed value $R M_{\mathrm{obs}}$ as:

$R M_{\text {intr }}=R M_{\text {obs }} \times\left(1+z_{\mathrm{F}}\right)^{2}$.

For the radio galaxies, it is most probable that the Faraday screen that produces the $R M$ is located at the same redshift of the radio sources. We can exclude a Galactic origin for the Faraday rotation since at latitudes $b>20^{\circ}$ the contribution of the Galactic screen is of order of $10 \mathrm{rad} \mathrm{m}{ }^{-2}$ with $R M$ gradients of $<<10 \mathrm{rad} \mathrm{m}^{-2}$ over $1^{\prime \prime}$ (e.g. Leahy 1987) while we observe much larger gradients between the two (or more) hot-spots of each radio source. For example in the radio galaxy $1202+527$ there is a gradient of more than $300 \mathrm{rad} \mathrm{m}^{-2}$ with a sign reversal, over only $4^{\prime \prime}$. Contribution from intervening structures such as galaxies and clusters, which have $\mu \mathrm{G}$ magnetic fields correlated over kpc or 10ns of kpc scales, or absorption line systems, can be also ruled out on the basis of small probability (e.g. Athreya et al. 1998).

Therefore if the $R M$ screen is in the vicinity of the radio source, the values listed in Table 3 have to be multiplied by a factor of 10 to 20 (depending on redshift), implying $R M \mathrm{~s}$ of the order of several $100 \mathrm{rad} \mathrm{m}^{-2}$ for most sources and in excess of $1000 \mathrm{rad} \mathrm{m}^{-2}$ for 8 sources, with a maximum of $3100 \mathrm{rad} \mathrm{m}^{-2}$ for the radio source $0930+389$.

Carilli et al. (1994) were the first to point out the existence of very large Faraday rotation in HzRGs. In the previous VLA observational study similar to this, they found that about $20 \%$ of radio galaxies had intrinsic $R M$ in excess with $1000 \mathrm{rad} \mathrm{m}^{-2}$, while Athreya et al. (1998) found high $R M$ in 4 out of 15 radio galaxies. Both these results are in agreement with the results from the present sample.

At low redshift most powerful radio galaxies show

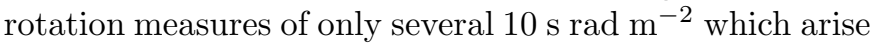
in the interstellar medium (ISM) of our Galaxy. However few radio galaxies have a $R M$ in excess of $1000 \mathrm{rad} \mathrm{m}^{-2}$ (Taylor et al. 1994): these are either compact (sub galactic in size) radio sources or are radio galaxies located in X-rays cooling flow clusters. In fact Taylor et al. (1994) found that the strength of the cooling flow (or alternatively the core density of the cluster) directly correlates with the rotation measure. An important point is that the above relation is independent of radio source luminosity and morphological class: since FRI and FRII type galaxies have very different physical interactions, this suggests that the Faraday rotation is most likely a probe of cluster properties and not radio source properties. The physical conclusion is that for those sources located in clusters, the $R M$ arises in the dense $\mathrm{X}$-ray emitting gas which is substantially magnetized. 

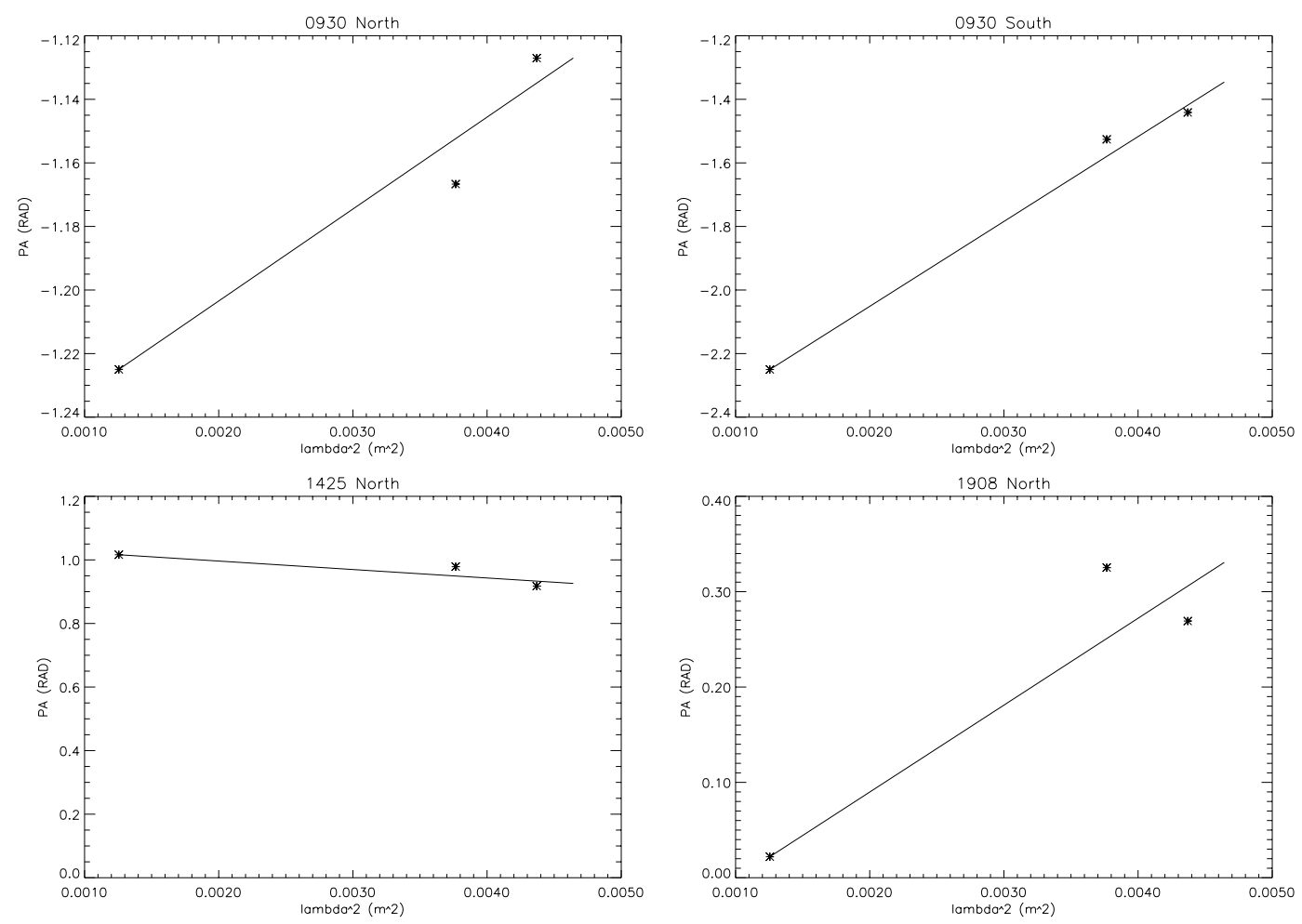

Fig. 3. Polarization position angles versus $\lambda^{2}$ (in $\mathrm{cm}$ ) for the lobes of some HzRGs in our sample: upper panels are the components of 0930 north (left) and south (right). The lower panels are 1425 north (left) and 1988 north (right)

5.4.1. Does Faraday rotation depend on radio source properties?

To determine the nature of the Faraday screen at high redshift we first investigated whether the Faraday rotation is significantly correlated with the radio source morphology or other properties. As mentioned in the previous section, at low redshift Faraday rotation does not depend on radio source morphology or luminosity: this might not be the case at high redshift, where it is believed that the interactions of the radio jets with the host galaxies and the ambient medium are much stronger, as shown for example by the increasing distorted morphology of radio sources discussed in Sect. 5.2.

From the combined sample of about 70 radio galaxies at $z>2$ with homogeneous high resolution radio polarimetric VLA observations (Carilli et al. 1994 and 1997; Athreya et al. 1998 and this paper), we selected all HzRGs with observed $R M$ larger than $40 \mathrm{rad} \mathrm{m}^{-2}$ (lower observed values might be effected by relatively larger errors). The total number of selected HzRGs is 37, of which 23 have intrinsic $R M$ larger than $1000 \mathrm{rad} \mathrm{m}^{-2}$.

To characterize a radio source we used the following parameters: (a) the monochromatic power at a rest-frame frequency of $178 \mathrm{MHz}$; (b) the total extent of the radio source; (c) the integrated spectral index between 8.2 and $4.5 \mathrm{GHz}$ (observed frequencies); (d) the core fraction at a rest-frame frequency of $20 \mathrm{GHz}$; (e) the number of hot- spots defined as the number of separate emission peaks in the $8.2 \mathrm{GHz}$ maps and (f) the bending angle. Assuming orientation unification models (e.g. Barthel 1989) the core fraction allows us study line-of sight effects, knowing that radio sources in general have a smaller core fraction (typically $1-2 \%$ ) than radio loud quasars and therefore those with higher core fractions will be in the transition zone between radio galaxies and quasars. The total length of the source is used to see if Faraday rotation is related to the inner region of the galaxy, and hence is shown only by radio sources whose size is comparable to the galaxy, like the CSS sources that have sizes of less than $20 \mathrm{kpc}$, or is a larger scale effect. Finally parameters such as the number of hot-spot and the bending angle, give us information on the distortion of the radio sources, which is probably related to the density of the near environment of the object (e.g. Barthel \& Miley 1988).

In Fig. 4 we present the results of these investigations: we do not see any significant dependence of the Faraday rotation on any of above parameters. Therefore we conclude that, at high redshift, Faraday rotation is independent of radio source luminosity and morphology, and is probably not the probe of radio sources properties but of their environment.

Drawing the analogy with lower redshift sources having extreme $R M$, the conclusion is that also the HzRGs with large Faraday rotation might reside in dense, protocluster environment. Indeed selecting the HzRGs with 

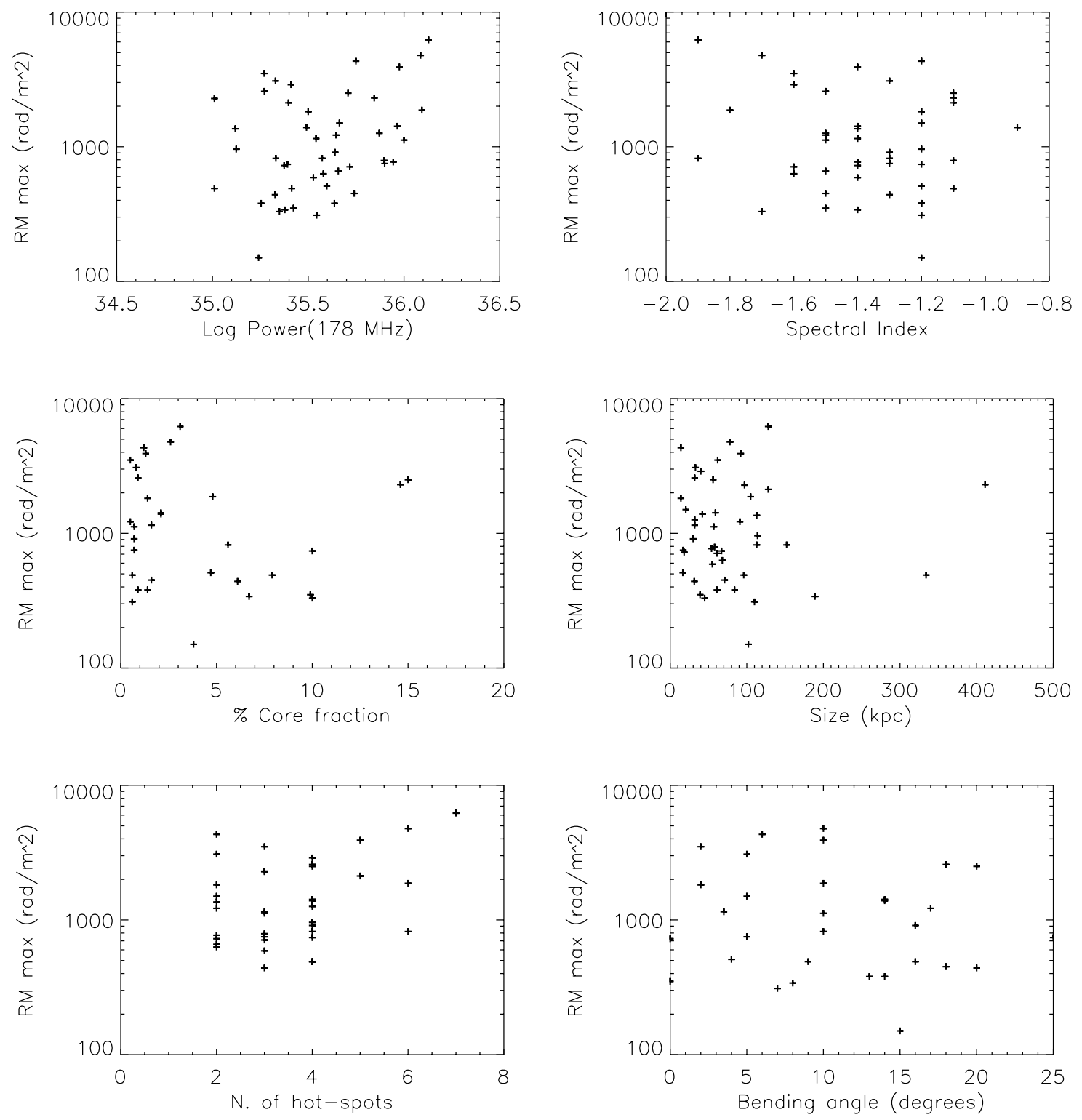

Fig. 4. The maximum Faraday rotation observed in high redshift radio galaxies, plotted against different energetic and morphological properties of the radio sources (see text)

highest known Faraday rotation $\left(6600 \mathrm{rad} \mathrm{m}^{-2}\right)$ our group has detected possibly extended X-ray emission around the radio galaxy $1138-262$ at a redshift of 2.2 (Carilli et al. 1998). If confirmed (time has been allocated at the Chandra X-ray observatory to re-observe the source with a much higher spatial resolution), this would be the highest redshift X-ray cluster known to date.

Including the new sources presented in this paper, there are now a total of $23 \mathrm{HzRG}$ sith Faraday rotation exceeding $1000 \mathrm{rad} \mathrm{m}^{-2}$, with redshift ranging from 2.2 to 3.8 (for a complete list see Pentericci 1999). Of these, 3 are CSS sources for which the origin of the Faraday rota- tion could be the local ISM, given that the radio sources are completely embedded within the host galaxies. The remaining 20 are excellent targets when searching for the most distant (proto)clusters in the early Universe.

\subsubsection{Comparison with low redshift radio galaxies}

To investigate whether the fraction of galaxies with extreme $R M$ changes as a function of redshift, we gathered data from the literature for a sample of powerful radio galaxies at low and intermediate redshift to be compared to our sample. We selected all objects from the $3 \mathrm{CR}$ 


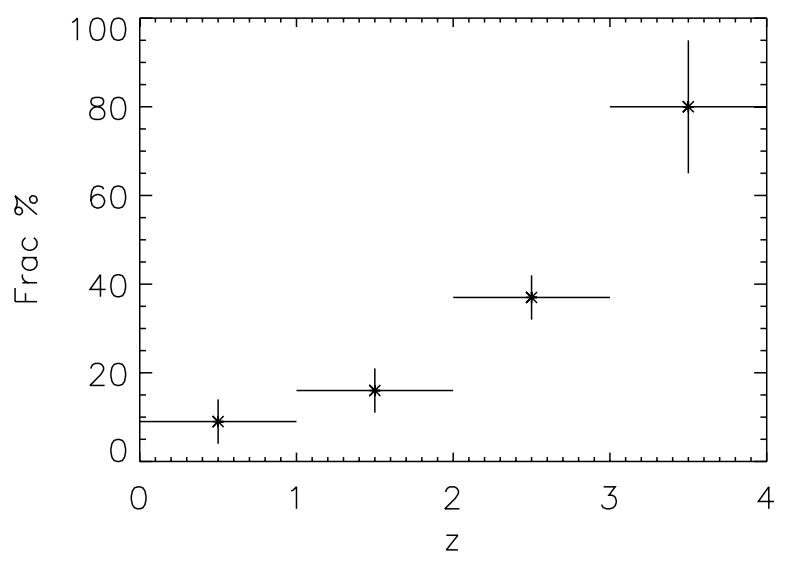

Fig. 5. The fraction of powerful radio galaxies with Faraday rotation in excess of $1000 \mathrm{rad} \mathrm{m}^{-2}$ as a function of redshift; the horizontal error bars indicate the redshift range for each bin

catalogue (Laing et al. 1983) having a monochromatic power at $178 \mathrm{MHz}$ (rest-frame frequency) of $\log P_{178}>$ 34.8 (with $P_{178}$ in units of $\mathrm{erg} \mathrm{s}^{-1} \mathrm{~Hz}^{-1}$ ) an arbitrary value chosen in order to have relatively powerful radio sources, with still a considerable number of low redshift sources. We added these sources to the sample of radio galaxies at $z \geq 2$. We then eliminated the CSS sources from the resulting list: in these galaxies the radio source is generally completely embedded within the host galaxy, so the $R M$ is due to the local magnetized ISM. CSS sources generally have very high Faraday rotation, therefore if distribution of CSS sources with redshift is different from that of large radio sources (e.g. O'Dea 1998), this will modify the final result. The final sample contains $\sim 90$ radio galaxies spanning a redshift range from $z=0.015$ (Cygnus A) to $z=3.8$ (4C41.17).

We then searched for Faraday rotation measurements for the 3CR galaxies: all of the values were taken from Tabara \& Inoue (1980) and Inoue et al. (1995), with the exception of the radio galaxy Cygnus $\mathrm{A}$ for which much more detailed studies have been carried out (Carilli \& Barthel 1996). The results are presented in Fig. 5, where we plot the fraction of galaxies with $R M \mathrm{~s}$ above $1000 \mathrm{rad} \mathrm{m}^{-2}$ as a function of redshift, for four redshift intervals. The fraction of galaxies with $R M \mathrm{~s}$ above the line clearly increases with $z: 9 \pm 4 \%$ at $z<1,16 \pm 5 \%$ at $1<z<2,37 \pm 5 \%$ at $2<z<3$ and $80 \pm 15 \%$ at $z>3$ (although in the last redshift bin there are only 6 galaxies and therefore the statistics is very low).

Some care has to be taken when considering this result, given the following limitations to our analysis: (i) first, most high redshift radio galaxies were found by selecting ultra steep spectrum radio sources (radio spectral index $\alpha<-1$, where $S_{\nu} \propto \nu^{\alpha}$, with $S_{\nu}$ the flux density and $\nu$ the frequency). This means that if Faraday rotation depends on spectral index (but in the previous section we showed that this is not the case, at least for a limited range of $\alpha$ ), then our results will be biased; (ii) the quality of the different radio observations is not matched in resolution: since one always measures the average $R M$ within the beam size, having a large beam size implies measuring lower values of $R M$; (iii) finally, and most important, the various sets of observations have different wavelength coverage, which determines the highest and lowest values of $R M$ observable. Note that the resolution effect (ii) would tend to decrease the correlation found, since at higher redshift the beam size in kpc will tend to be larger. Furthermore, within our sample (redshift $z>2$ ), the physical resolution is nearly constant, but we still detect the correlation between redshift and Faraday rotation.

Despite the above limitations, we regard the effect apparent in Fig. 5 to be real, namely that the fraction of powerful radio galaxies with high Faraday rotation increases with redshift. If high Faraday rotation is an indication of dense environment, this result is consistent with the fact that the average environment of powerful radio sources becomes denser with increasing redshift (e.g. Hill \& Lilly 1991). At low redshift most powerful radio sources (FRII type) reside in sparse environment with few exceptions (e.g. Cygnus A), while at earlier epochs more and more radio galaxies reside in dense environments (e.g. Roche et al. 1998).

\section{Conclusions}

We have presented high resolution multi-frequency radio polarimetric observations of a sample of 27 high redshift radio galaxies. Maps of the sources and the fundamental parameters of the observations were presented. This, together with previous samples makes now an extended data base from which the relation between basic properties can be studied. The main results are the following:

- We detect radio cores in about half of the sample. The cores often have steep spectra $(\alpha<-1)$. The core fractions depend only weakly on radio sources size, contrary to the predictions of radio source evolutionary models. The median core fraction is larger than that of matched-luminosity $3 \mathrm{CR}$ radio galaxies at redshift $\sim 1$.

- We have shown that high redshift radio galaxies tend to be more distorted than at low redshift. This implies a larger density of the external medium in which they reside.

- We have discovered 8 new radio galaxies with very high Faraday rotation and large gradients between the different components. Given that the Faraday rotation properties do not depend on radio sources parameters such as power, total size, distortion etc., our interpretation is that these sources reside in very high density environments, possibly proto-clusters. We also find that the fraction of powerful radio galaxies with extreme 
Faraday rotation increases with redshift, in agreement with the change of their average environment with cosmic epoch.

Acknowledgements. The National Radio Astronomy Observatory is operated by Associated Univ. under contract with the National Science Foundation. This research has made use of the NASA/IPAC Extragalactic Database (NED) which is operated by the Jet Propulsion Laboratory, California Institute of Technology, under contract with the National Aeronautics and Space Administration.

\section{References}

Athreya R., 1996, Ph.D. Thesis, Tata Institute of Fundamental Research

Athreya R.M., Kapahi V.K., McCarthy P.J., van Breugel W., 1997, MNRAS 289, 525

Athreya R.M., Kapahi V.K., McCarthy P.J., van Breugel W., 1998, A\&A 329, 809

Barthel P.D., 1989, ApJ 336, 606

Barthel P.D., Miley G.K., 1988, Nat 333, 319

Best P.N., Longair M.S., Röttgering J.H.A., 1997, MNRAS 292, 758

Best P.N., Eales S.A., Longair M.S., Rawlings S., Röttgering H.J.A., 1999, MNRAS 303, 616

Blundell K.M., Rawlings S., Eales S.A., Taylor G.B., Bradley A.D., 1998, MNRAS 295, 265

Blundell K.M., Rawlings S., Willott C.J., 1999, AJ 117, 677

Carilli C.L., Barthel P.D., 1996, ARA\&A 7, 1

Carilli C.L., Harris D., Pentericci L., et al., 1998, ApJ 494L, 143

Carilli C.L., Owen F.N., Harris D.E., 1994, AJ 107, 480

Carilli C.L., Röttgering H., van Ojik R., Miley G.K., van Breugel W.J.M., 1997, ApJS 109, 1

Chambers K.C., Miley G.K., van Breugel W.J.M., Huang J.S., 1996, ApJS 106, 215

De Breuck C., van Breugel W., Minniti D., et al., 1999, A\&A 352, L51

De Breuck C., et al., 1998, Observational Cosmology with the New Radio Surveys, p. 185
Eales S., Rawlings S., Law-Green D., Gotter G., Lacy M., 1997, MNRAS 291, 593

Eales S., 1998, in "The most distant radio galaxies", Röttgering H., Best P. and Lehnert M. (eds.), p. 33

Fanti R., Fanti C., Schilizzi R.T., et al., 1990, A\&A 231, 333

Kaiser C.R., Dennett-Thorpe J., Alexander P., 1997, MNRAS 292, 723

Kaphai V.K., 1990, in "Parsec scale radio jets", Zensus J., Pearson J. (eds.), p. 305

Hill G.J., Lilly S.J., 1991, ApJ 367, 1

Inoue M., Tabara H., Kato T., Aizu K., 1995, PASJ 47, 725

Laing R.A., Riley J.M., Longair M.S., 1983, MNRAS 204, 151

Leahy J.P., 1987, MNRAS 226, 433

Lonsdale C.J., Barthel P.D., Miley G.K., 1993, ApJS 87, 63

McCarthy P., Kaphai V., van Breugel W., et al., 1996, ApJS 107, 19

McCarthy P.J., van Breugel W., Kapahi V.K., 1991, ApJ 371, 478

Miley G.K., 1980, ARA\&A 18, 165

Muxlow T.W.B., Garrington S.T., 1991, in Hughes P.A. (ed.), "Beams and Jets in Astrophysics". Cambridge University Press Cambridge, p. 232

Neeser M.J., Eales S.A., Law-Green J.D., Leahy J.P., Rawlings S., 1995, ApJ 451, 76

Pentericci L., 1999, Ph.D. Thesis, University of Leiden

Pentericci L., Röttgering H., Miley G.K., et al., 1998, ApJ 504, 139

Pentericci L., Röttgering H., Miley G.K., et al., 1999, A\&A 341,329

Roche N., Eales S., Hippelein H., 1998, MNRAS 295, 946

Röttgering H.J.A., Lacy M., Miley G.K., Chambers K.C., Saunders R., 1994, A\&AS 108, 79

Saikia D.J., Salter C.J., 1988, ARA\&A 26, 93

Tabara H., Inoue M., 1980, A\&AS 39, 379

Taylor G.B., Barton E.J., Ge J., 1994, AJ 107, 1942

van Breugel W.J.M., De Breuck C., Stanford S.A., et al., 1999, ApJL 518, L61

van Breugel W.J.M., Stanford S.A., Spinrad H., Stern D., Graham J.R., 1998, ApJ 502, 614 

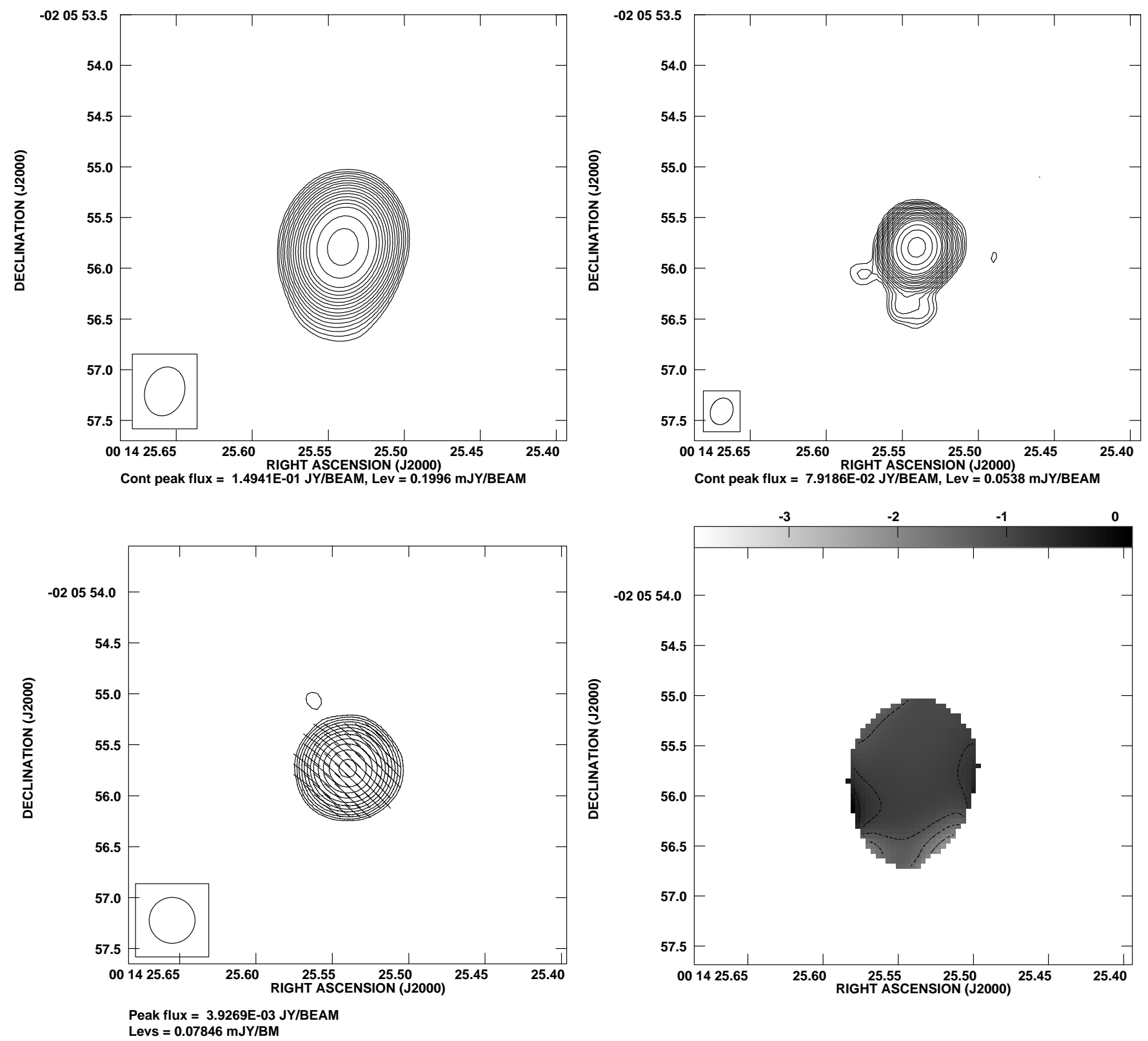

Fig. 6. Maps of the radio source $0011-023$ at redshift $z=2.08$. Upper left is the $4.5 \mathrm{GHz}$ total intensity map, having a FWHM of 0.5 arcsec, with the first contour at $0.2 \mathrm{mJy}$ beam $^{-1}$ and a peak surface brightness of $149 \mathrm{mJy} \mathrm{beam}{ }^{-1}$. Upper right is a contour map of total intensity at $8.2 \mathrm{GHz}$, with the first contour at $0.054 \mathrm{mJy}_{\text {beam }}{ }^{-1}$. Lower left is the map of polarized flux at $4.5 \mathrm{GHz}$, with the first contour $0.078 \mathrm{mJy}_{\text {beam }}{ }^{-1}$ and peak surface brightness of $39 \mathrm{mJy}$ beam $^{-1}$; superimposed are vectors representing the strenght and direction of the magnetic field. Lower right is a map of the spectral index calculated between the frequencies $4.5 \mathrm{GHz}$ and $8.2 \mathrm{GHz}$ 

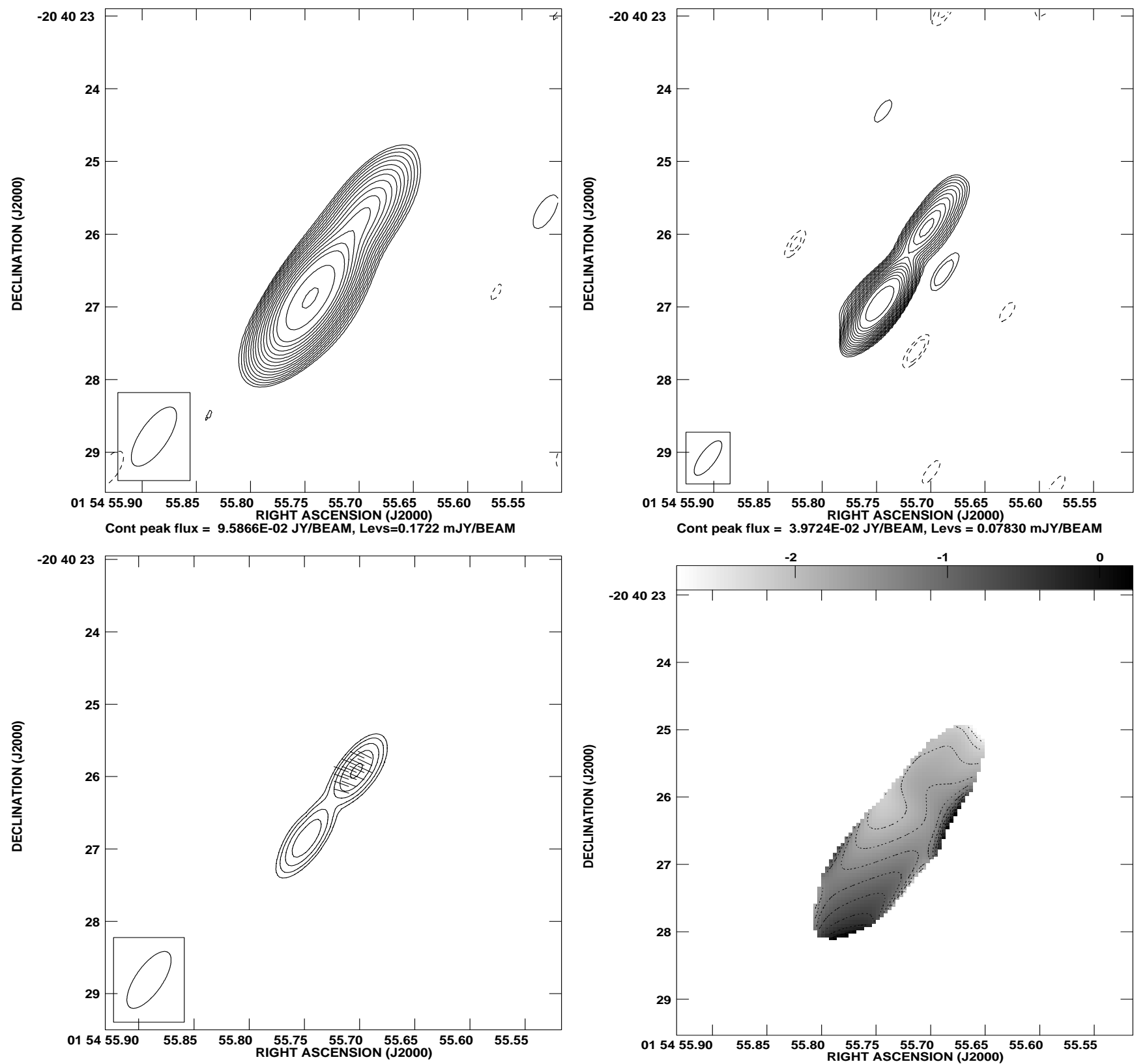

Peak flux $=4.7017 \mathrm{E}-04 \mathrm{JY} / \mathrm{BEAM}$, Levs $=0.1110 \mathrm{mJY} / \mathrm{BEAM}$

Fig. 7. Maps of the radio source $0152-209$ at redshift $z=1.89$. The sequence of figures is the same as in Fig. 6. The first contour level and the peak surface brightness are respectively $0.172 \mathrm{mJy}$ beam $^{-1}$ and $96 \mathrm{mJy} \mathrm{beam}^{-1}$ for the $4.5 \mathrm{GHz}$ map; $0.078 \mathrm{mJy}$ beam $^{-1}$ and $40 \mathrm{mJy}_{\text {beam }}{ }^{-1}$ for the $8.2 \mathrm{GHz}$ map; $0.11 \mathrm{mJy}_{\text {beam }}{ }^{-1}$ and $4.7 \mathrm{mJy}$ beam $^{-1}$ for the $4.7 \mathrm{GHz}$ polarized intensity map 

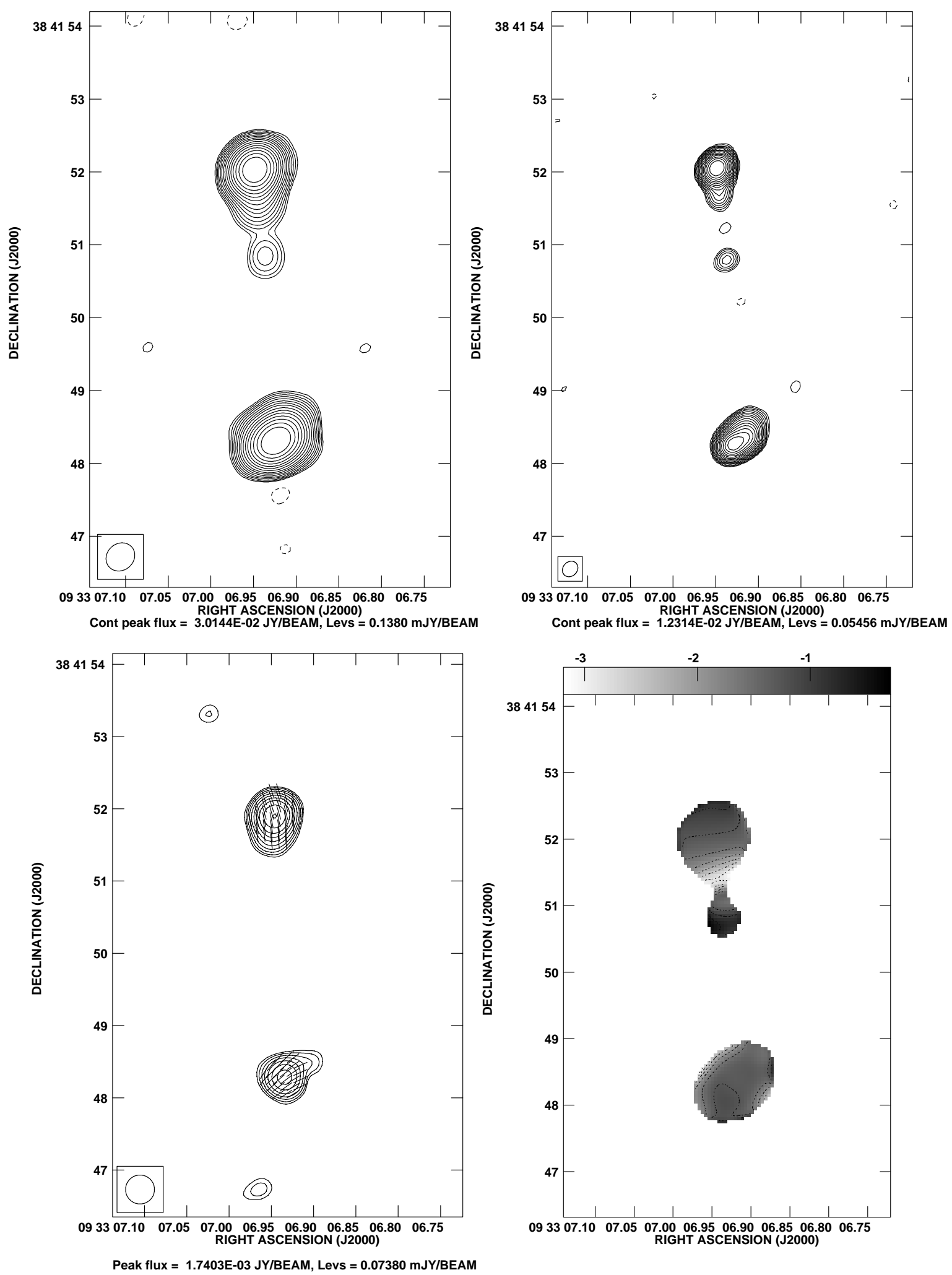

Fig. 8. Maps of the radio source $0930+389$ at redshift $z=2.395$. The sequence of figures is the same as in Fig. 6. The first contour level and the peak surface brightness are respectively $0.138 \mathrm{mJy} \mathrm{beam}^{-1}$ and $30 \mathrm{mJy} \mathrm{beam}^{-1}$ for the $4.5 \mathrm{GHz}$ map;

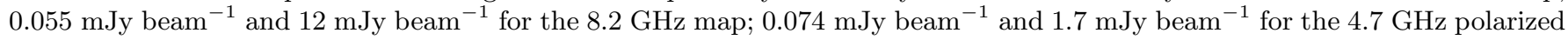
intensity map 

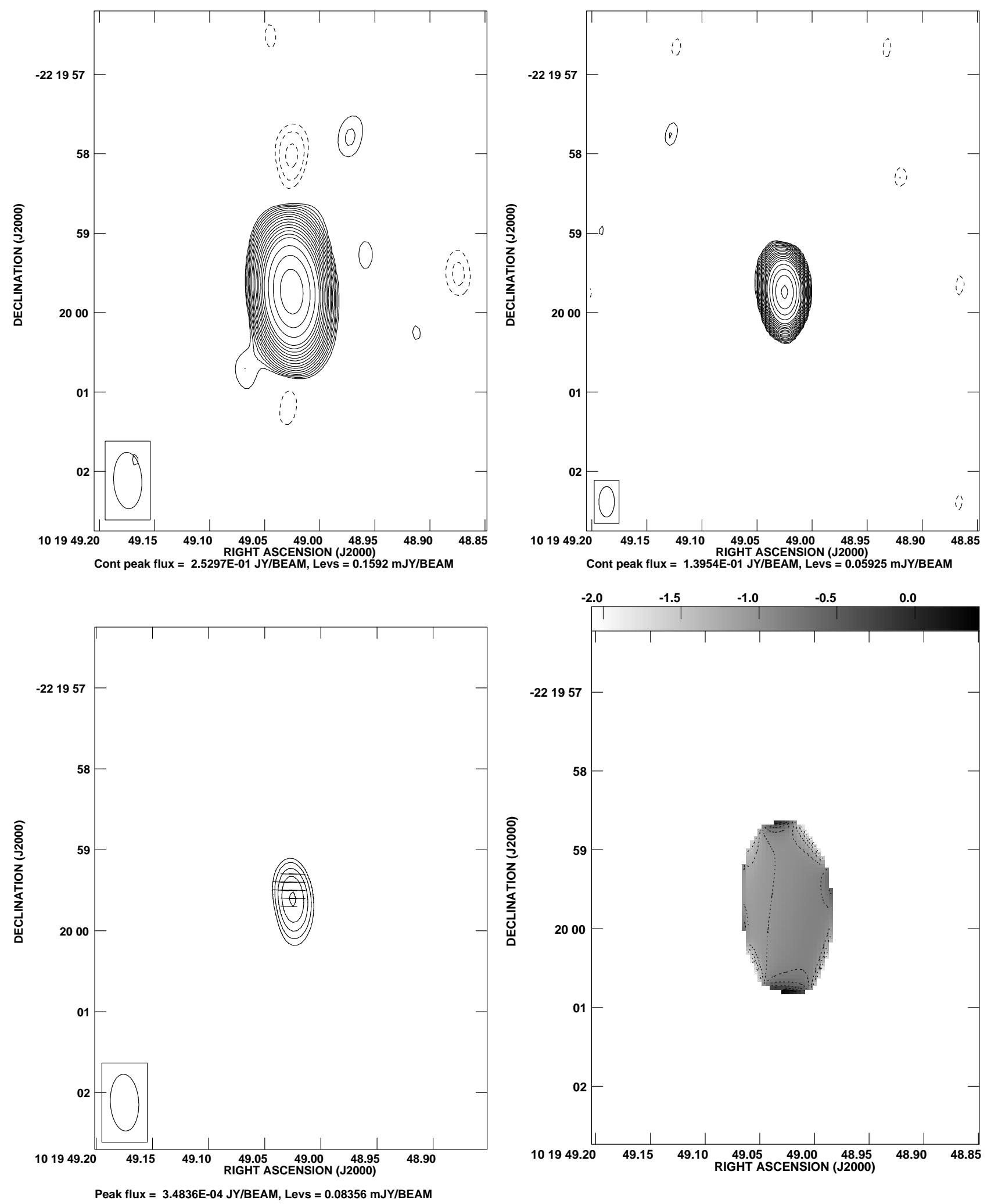

Fig. 9. Maps of the radio source $1017-220$ at redshift $z=1.77$. The sequence of figures is the same as in Fig. 6. The first contour level and the peak surface brightness are respectively $0.159 \mathrm{mJy}^{\mathrm{beam}}{ }^{-1}$ and $253 \mathrm{mJy} \mathrm{beam}^{-1}$ for the $4.5 \mathrm{GHz} \mathrm{map}$;

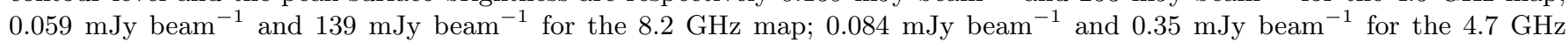
polarized intensity map 

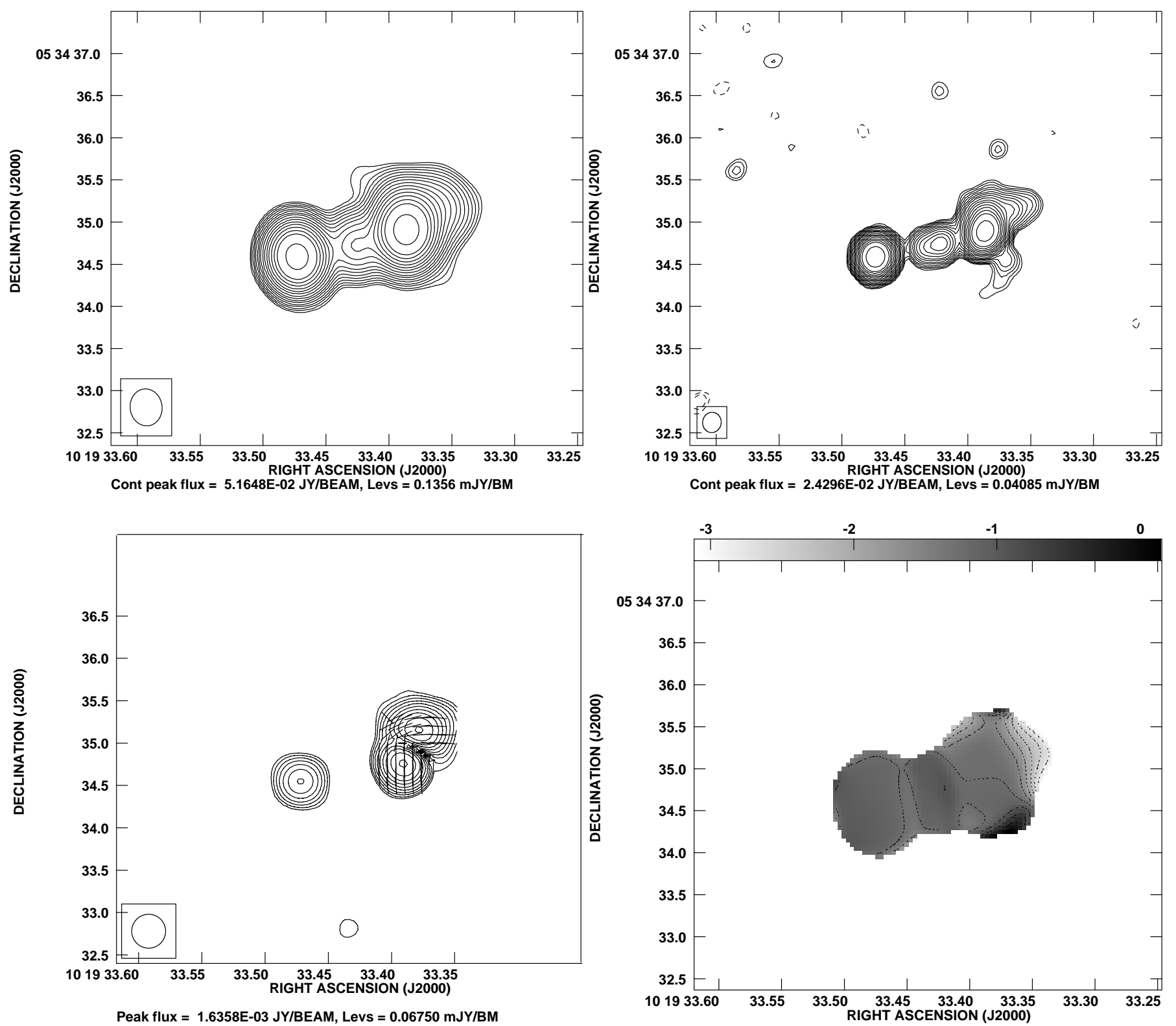

Fig. 10. Maps of the radio source $1019+0534$ at redshift $z=2.765$. The sequence of figures is the same as in Fig. 6. The first contour level and the peak surface brightness are respectively $0.136 \mathrm{mJy}^{\mathrm{beam}}{ }^{-1}$ and $52 \mathrm{mJy} \mathrm{beam}^{-1}$ for the $4.5 \mathrm{GHz}$ map;

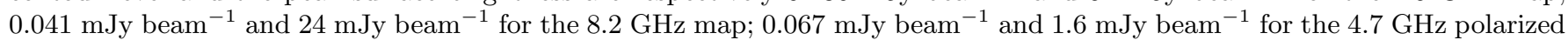
intensity map 

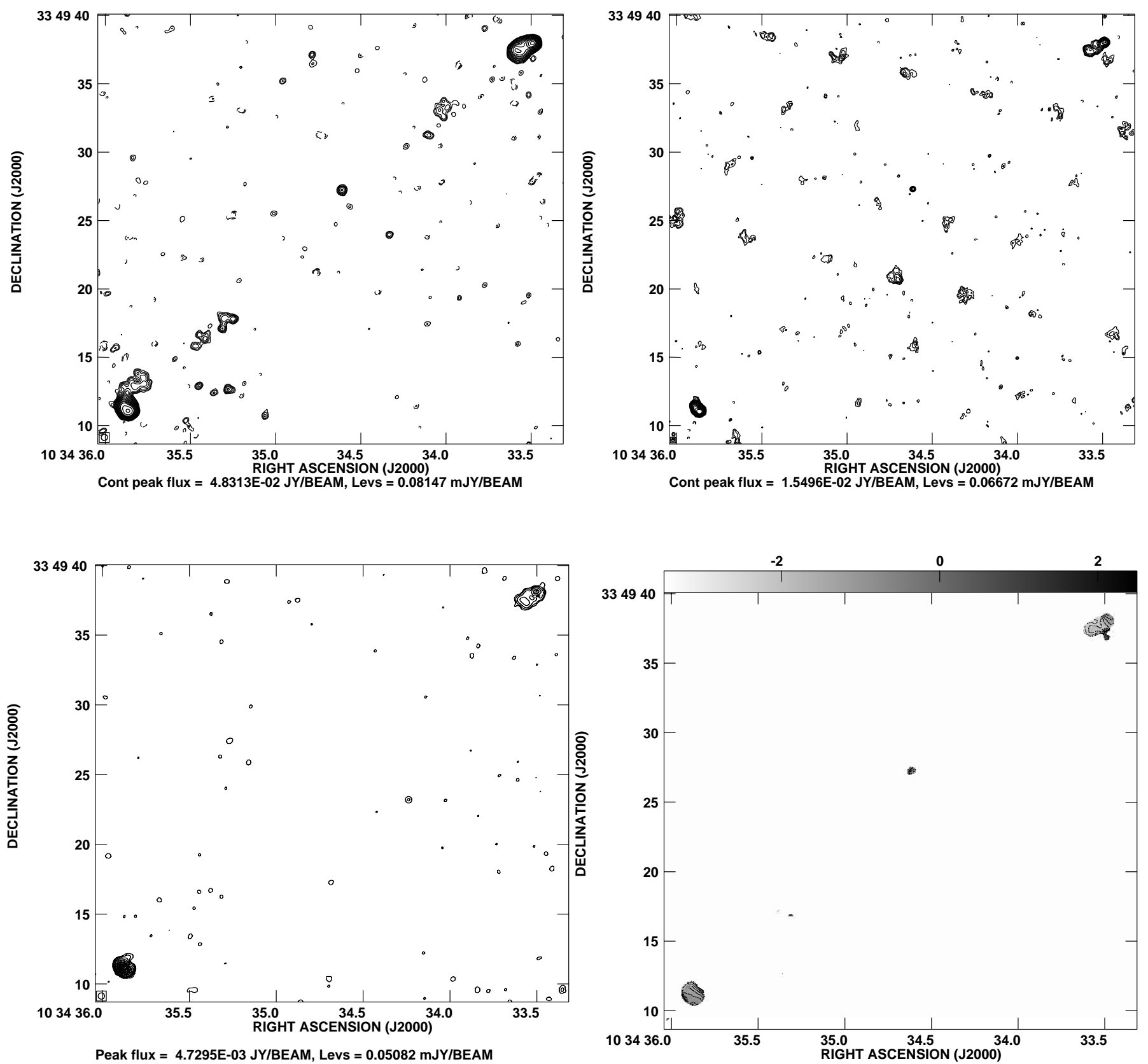

Fig. 11. Maps of the radio source $1031+34$ at redshift $z=2.10$. The sequence of figures is the same as in Fig. 6 . The first contour level and the peak surface brightness are respectively $0.081 \mathrm{mJy}^{-}$beam $^{-1}$ and $48 \mathrm{mJy}^{\mathrm{beam}}{ }^{-1}$ for the $4.5 \mathrm{GHz} \mathrm{map} ; 0.067 \mathrm{mJy}$ beam $^{-1}$ and $15 \mathrm{mJy}_{\text {beam }}^{-1}$ for the $8.2 \mathrm{GHz}$ map; $0.051 \mathrm{mJy}^{\mathrm{beam}}{ }^{-1}$ and $4.7 \mathrm{mJy}_{\text {beam }}{ }^{-1}$ for the $4.7 \mathrm{GHz}$ polarized intensity map 

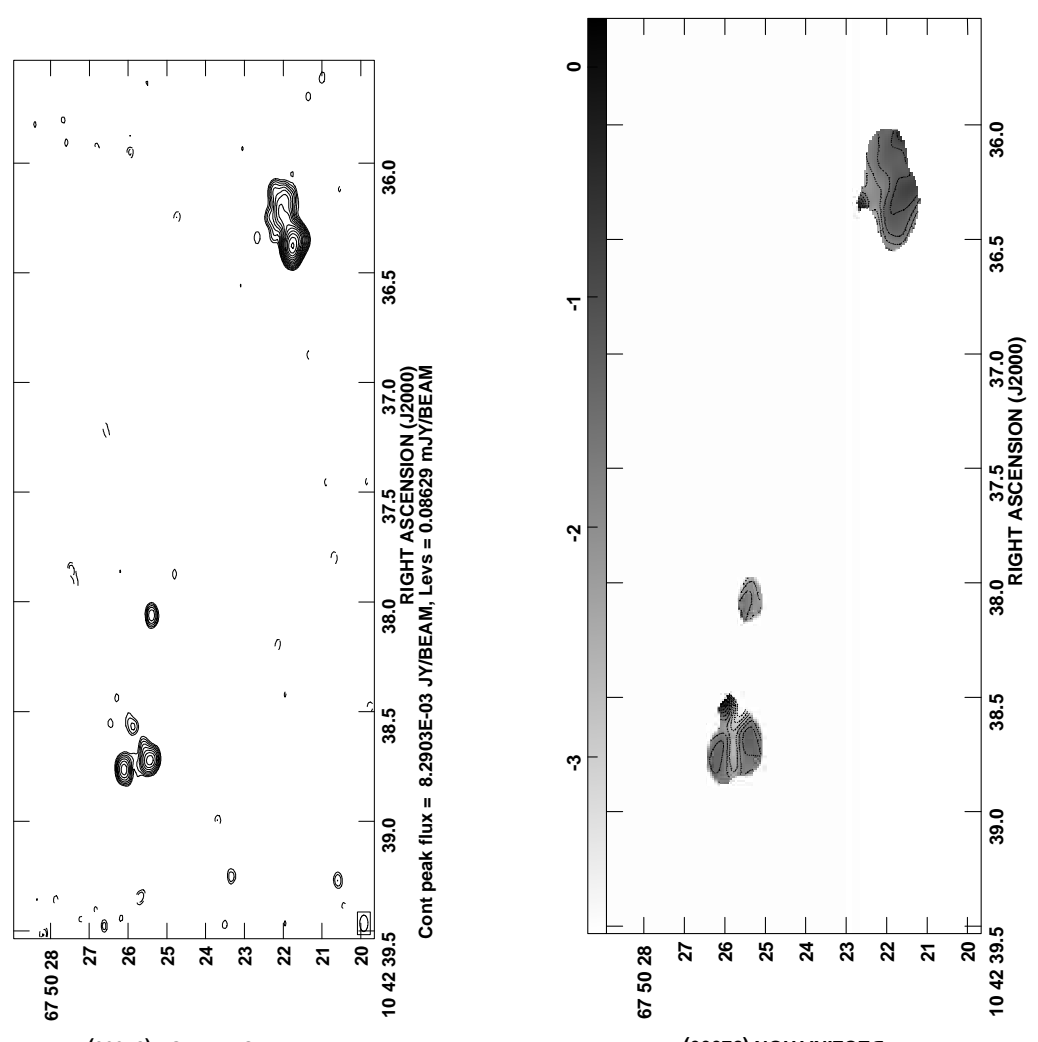

(0002) NOILVNI7OتG
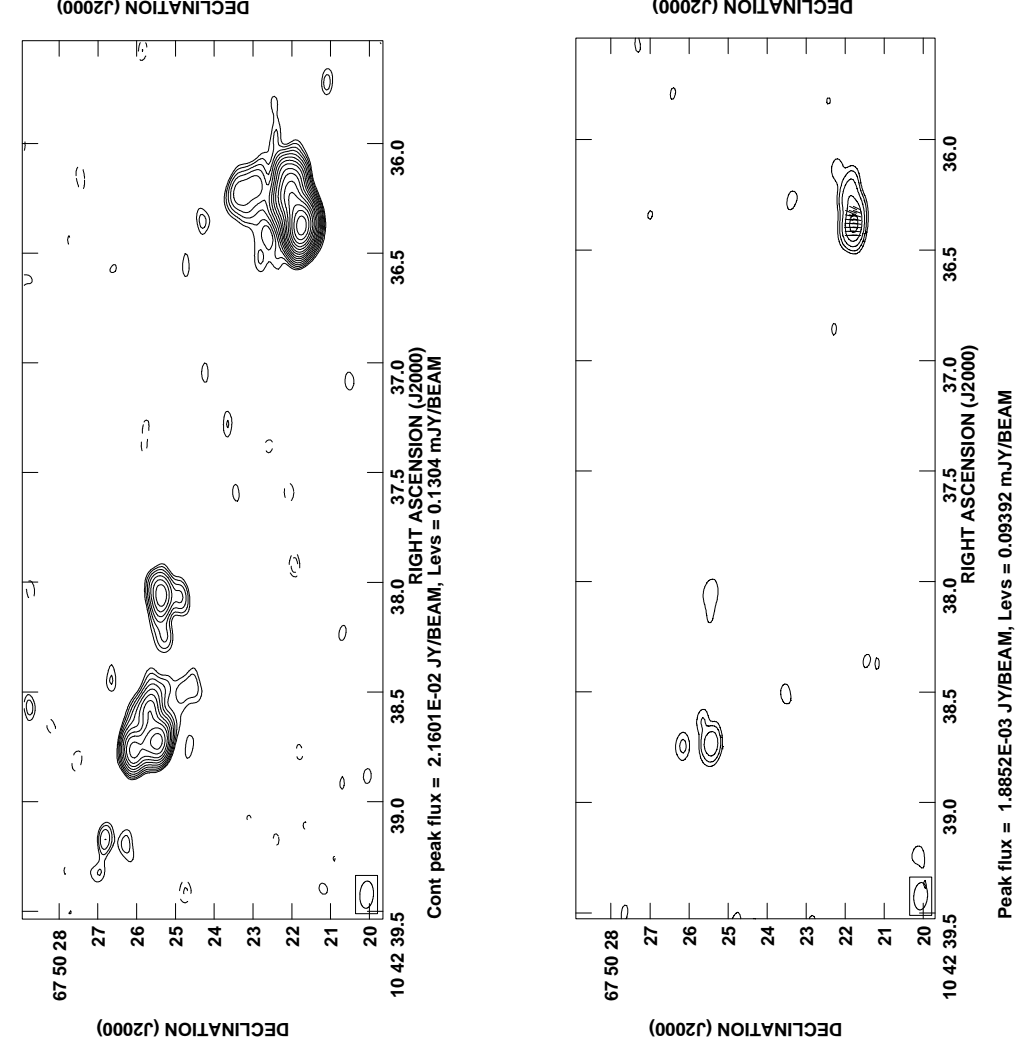

Fig. 12. Maps of the radio source $1039+681$ at redshift $z=2.53$. The sequence of figures is the same as in Fig. 6 . The first contour level and the peak surface brightness are respectively $0.134 \mathrm{mJy}$ beam $^{-1}$ and $22 \mathrm{mJy}$ beam $^{-1}$ for the $4.5 \mathrm{GHz} \mathrm{map}$; $0.086 \mathrm{mJy}$ beam $^{-1}$ and $8.3 \mathrm{mJy}_{\text {beam }}{ }^{-1}$ for the $8.2 \mathrm{GHz}$ map; $0.094 \mathrm{mJy}_{\text {beam }}^{-1}$ and $1.9 \mathrm{mJy}^{\mathrm{beam}}{ }^{-1}$ for the $4.7 \mathrm{GHz}$ polarized intensity map 

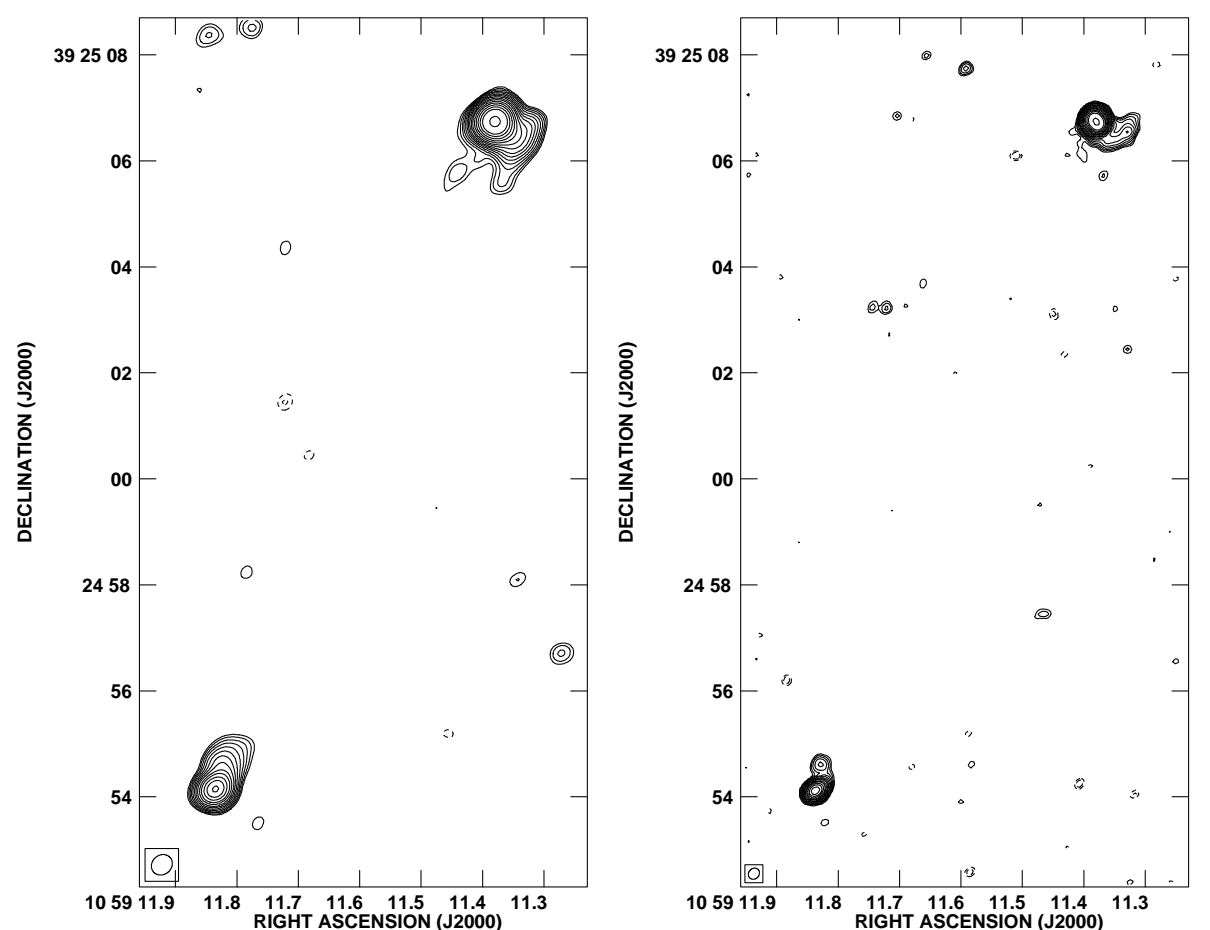

Cont peak flux $=4.2822 \mathrm{E}-02$ JY/BEAM, Levs $=0.1429 \mathrm{mJY} / \mathrm{BEAM}$ Cont peak fluX $=1.5712 \mathrm{E}-02 \mathrm{JY} / \mathrm{BEAM}$, Levs $=0.05293 \mathrm{mJY} / \mathrm{BE} A \mathrm{M}$
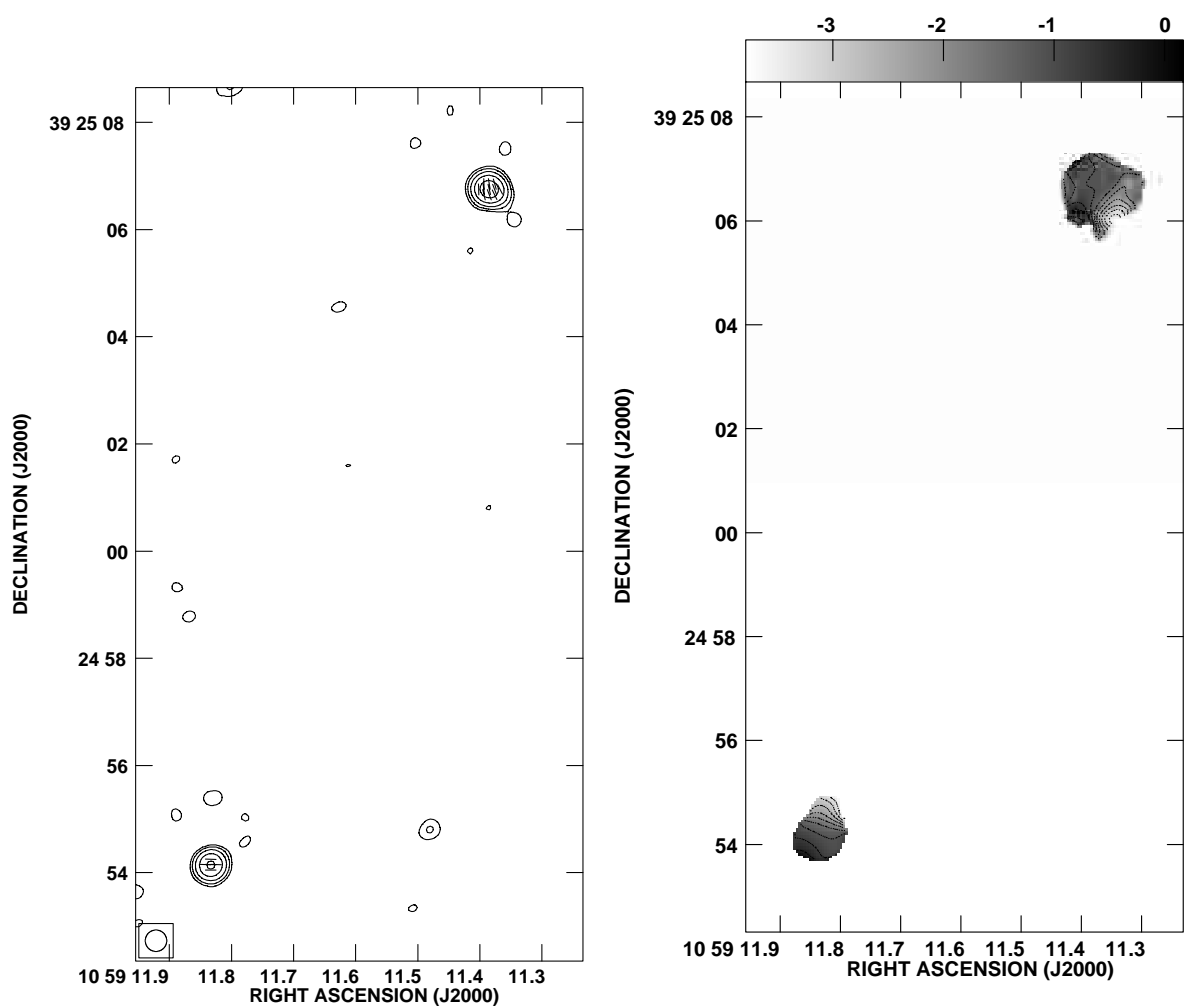

Peak flux $=1.8570 \mathrm{E}-03 \mathrm{JY} / \mathrm{BEAM}$, Levs $=0.07419 \mathrm{mJY} / \mathrm{BEAM}$

Fig. 13. Maps of the radio source $1056+39$ at redshift $z=2.171$. The sequence of figures is the same as in Fig. 6 . The first contour level and the peak surface brightness are respectively $0.143 \mathrm{mJy} \mathrm{beam}^{-1}$ and $43 \mathrm{mJy}$ beam $^{-1}$ for the $4.5 \mathrm{GHz}$ map;

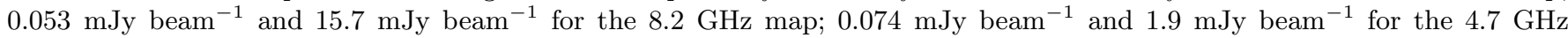
polarized intensity map 

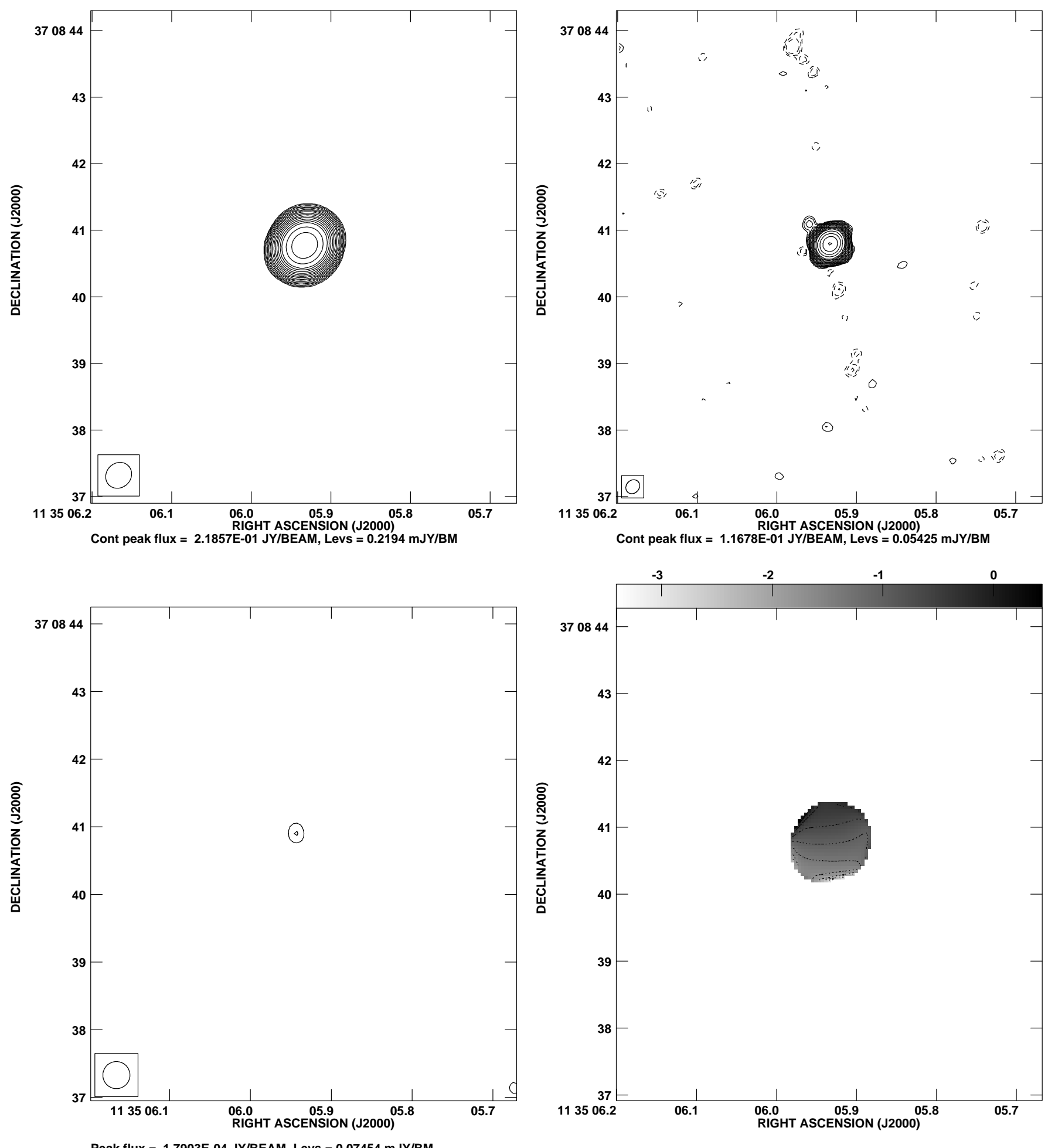

Fig. 14. Maps of the radio source $1132+37$ at redshift $z=2.88$. The sequence of figures is the same as in Fig. 6 . The first contour level and the peak surface brightness are respectively $0.22 \mathrm{mJy}^{\text {beam }}{ }^{-1}$ and $219 \mathrm{mJy} \mathrm{beam}^{-1}$ for the $4.5 \mathrm{GHz}$ map; $0.054 \mathrm{mJy}$ beam $^{-1}$ and $117 \mathrm{mJy}_{\text {beam }}^{-1}$ for the $8.2 \mathrm{GHz}$ map; $0.074 \mathrm{mJy}^{\text {beam }}{ }^{-1}$ and $0.2 \mathrm{mJy}_{\text {beam }}{ }^{-1}$ for the $4.7 \mathrm{GHz}$ polarized intensity map 

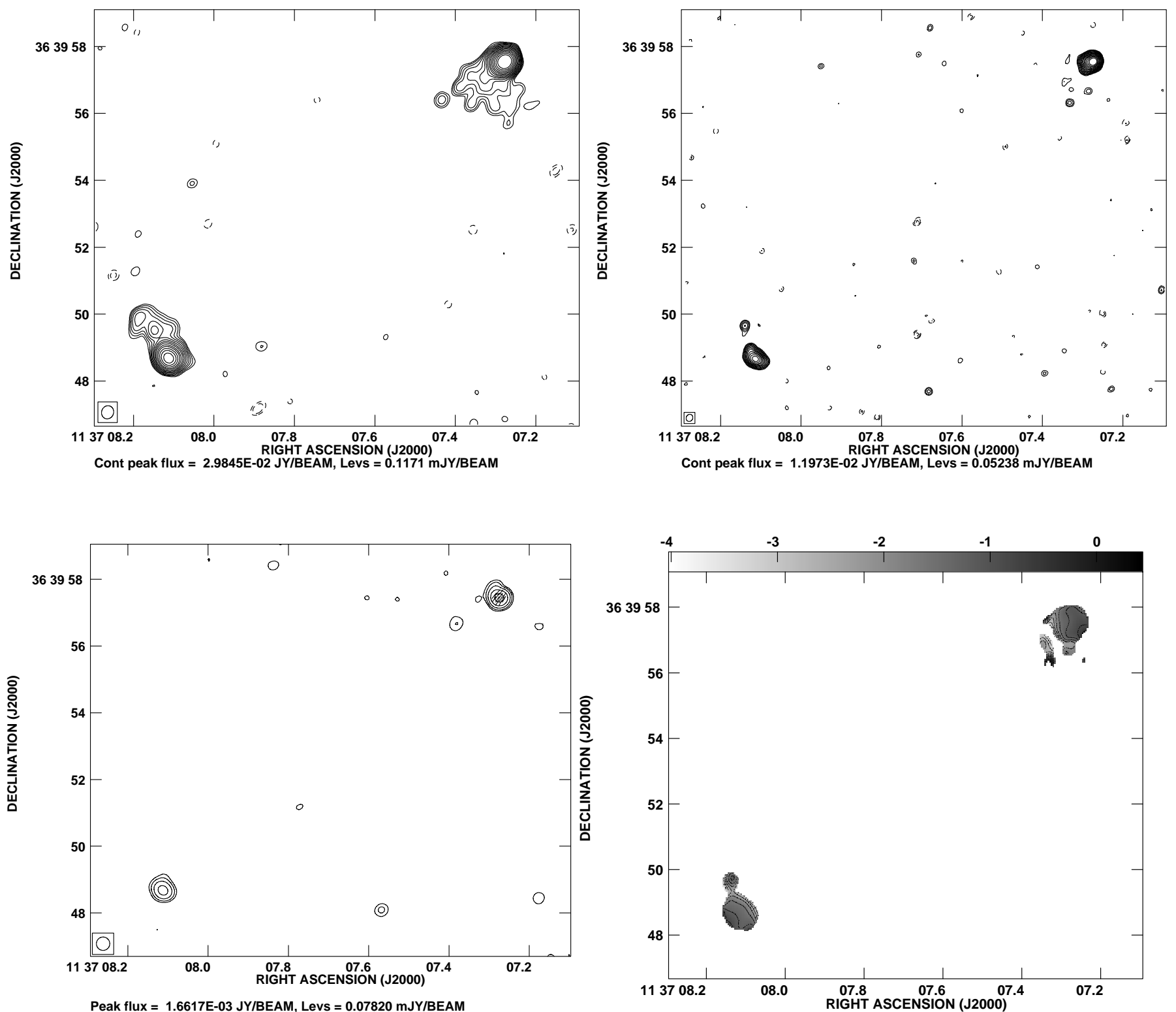

Fig. 15. Maps of the radio source $1134+369$ at redshift $z=2.12$. The sequence of figures is the same as in Fig. 6. The first contour level and the peak surface brightness are respectively $0.117 \mathrm{mJy}^{\text {beam }}{ }^{-1}$ and $30 \mathrm{mJy}$ beam $^{-1}$ for the $4.5 \mathrm{GHz}$ map;

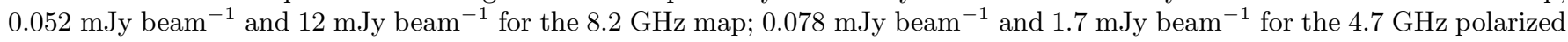
intensity map 

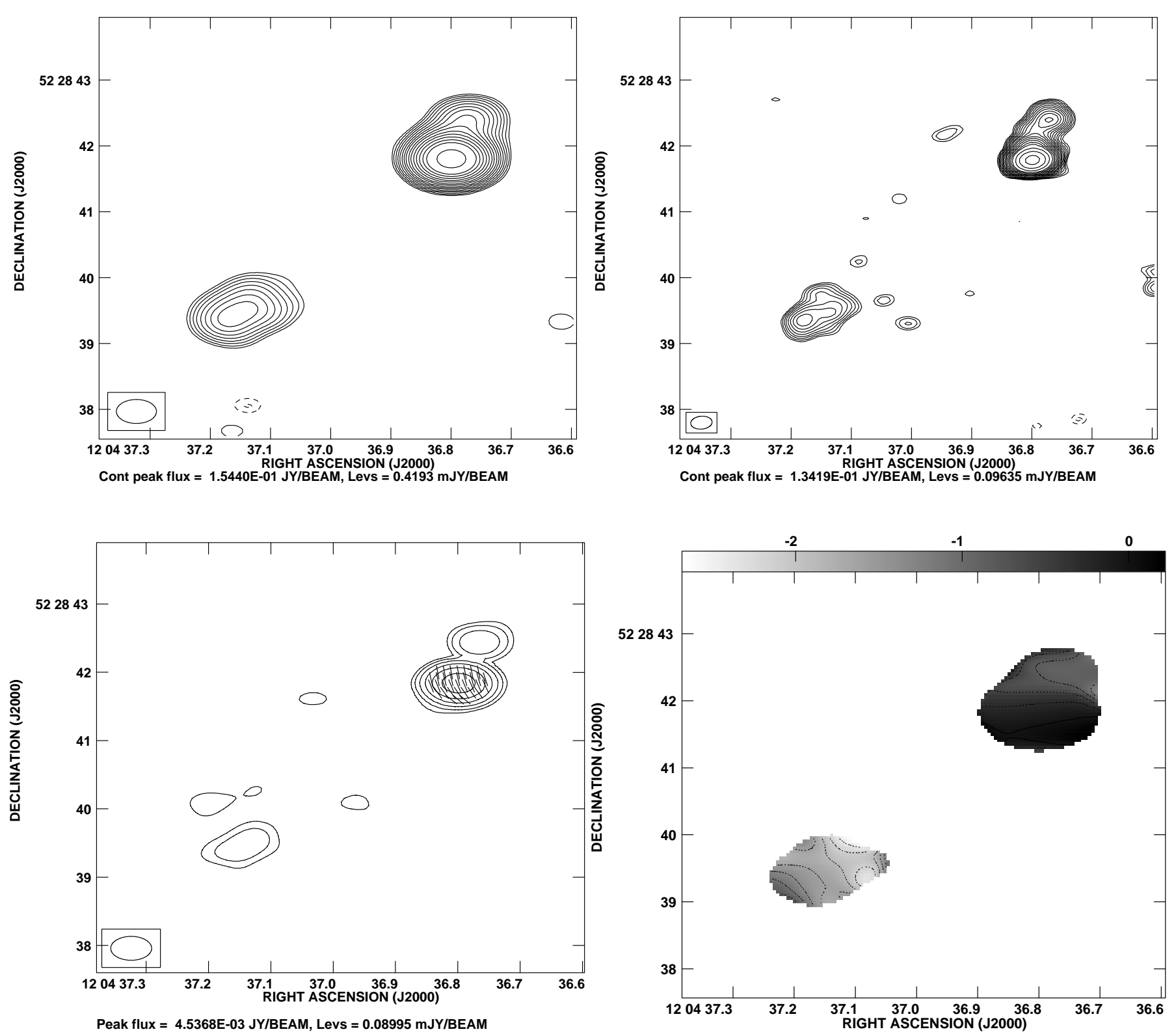

Fig. 16. Maps of the radio source $1202+527$ at redshift $z=2.73$. The sequence of figures is the same as in Fig. 6 . The first contour level and the peak surface brightness are respectively $0.42 \mathrm{mJy}_{\text {beam }}^{-1}$ and $154 \mathrm{mJy} \mathrm{beam}^{-1}$ for the $4.5 \mathrm{GHz} \mathrm{map}$; $0.096 \mathrm{mJy}$ beam $^{-1}$ and $134 \mathrm{mJy}_{\text {beam }}^{-1}$ for the $8.2 \mathrm{GHz}$ map; $0.090 \mathrm{mJy}_{\text {beam }}{ }^{-1}$ and $4.5 \mathrm{mJy}_{\text {beam }}{ }^{-1}$ for the $4.7 \mathrm{GHz}$ polarized intensity map 

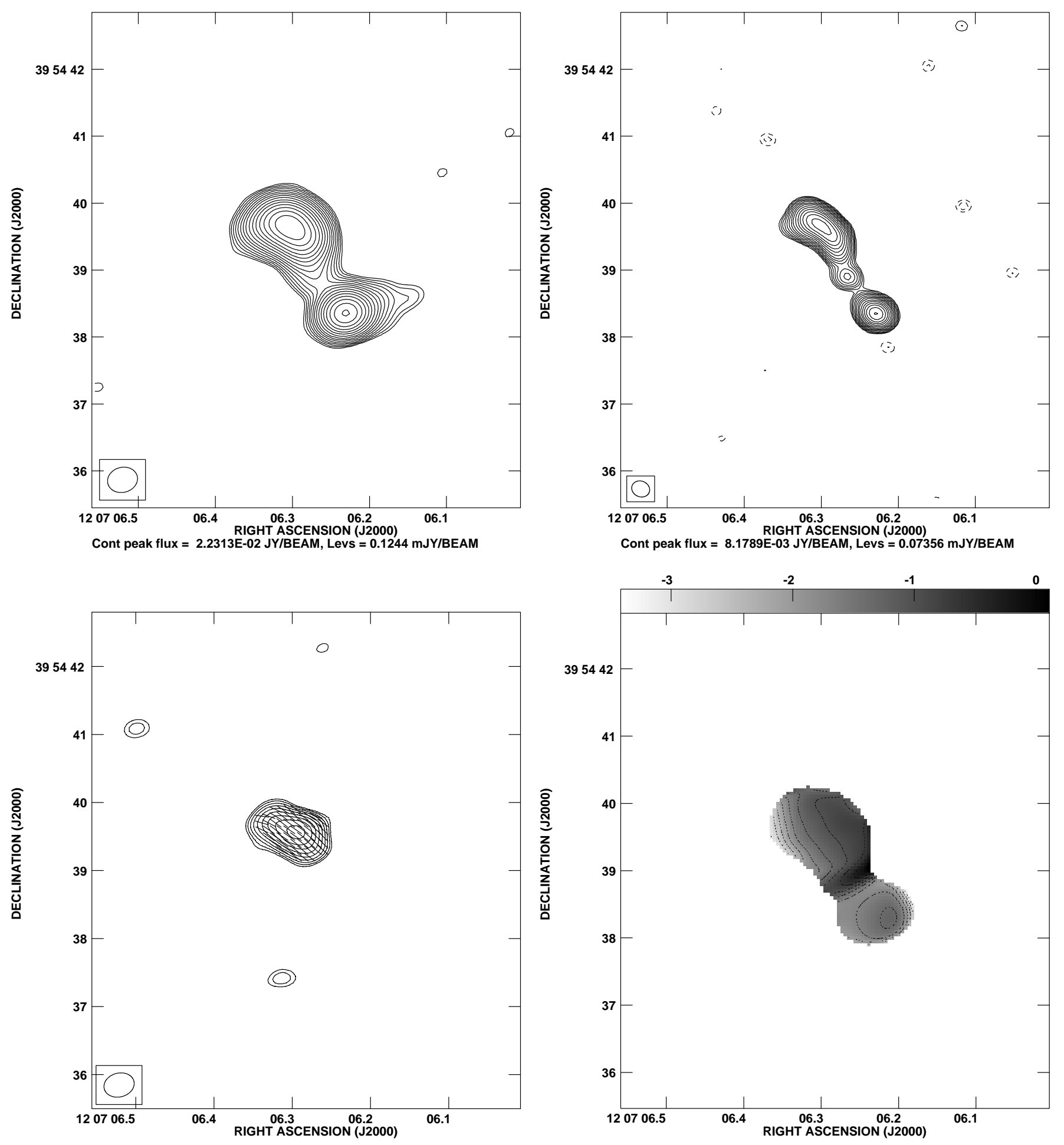

Fig. 17. Maps of the radio source $1204+401$ at redshift $z=2.066$. The sequence of figures is the same as in Fig. 6. The first contour level and the peak surface brightness are respectively $0.124 \mathrm{mJy}_{\text {beam }}{ }^{-1}$ and $22 \mathrm{mJy}$ beam $^{-1}$ for the $4.5 \mathrm{GHz}$ map; $0.062 \mathrm{mJy}_{\text {beam }}{ }^{-1}$ and $82 \mathrm{mJy}_{\text {beam }}{ }^{-1}$ for the $8.2 \mathrm{GHz}$ map; $0.077 \mathrm{mJy}_{\text {beam }}{ }^{-1}$ and $1.4 \mathrm{mJy}$ beam $^{-1}$ for the $4.7 \mathrm{GHz}$ polarized intensity map 

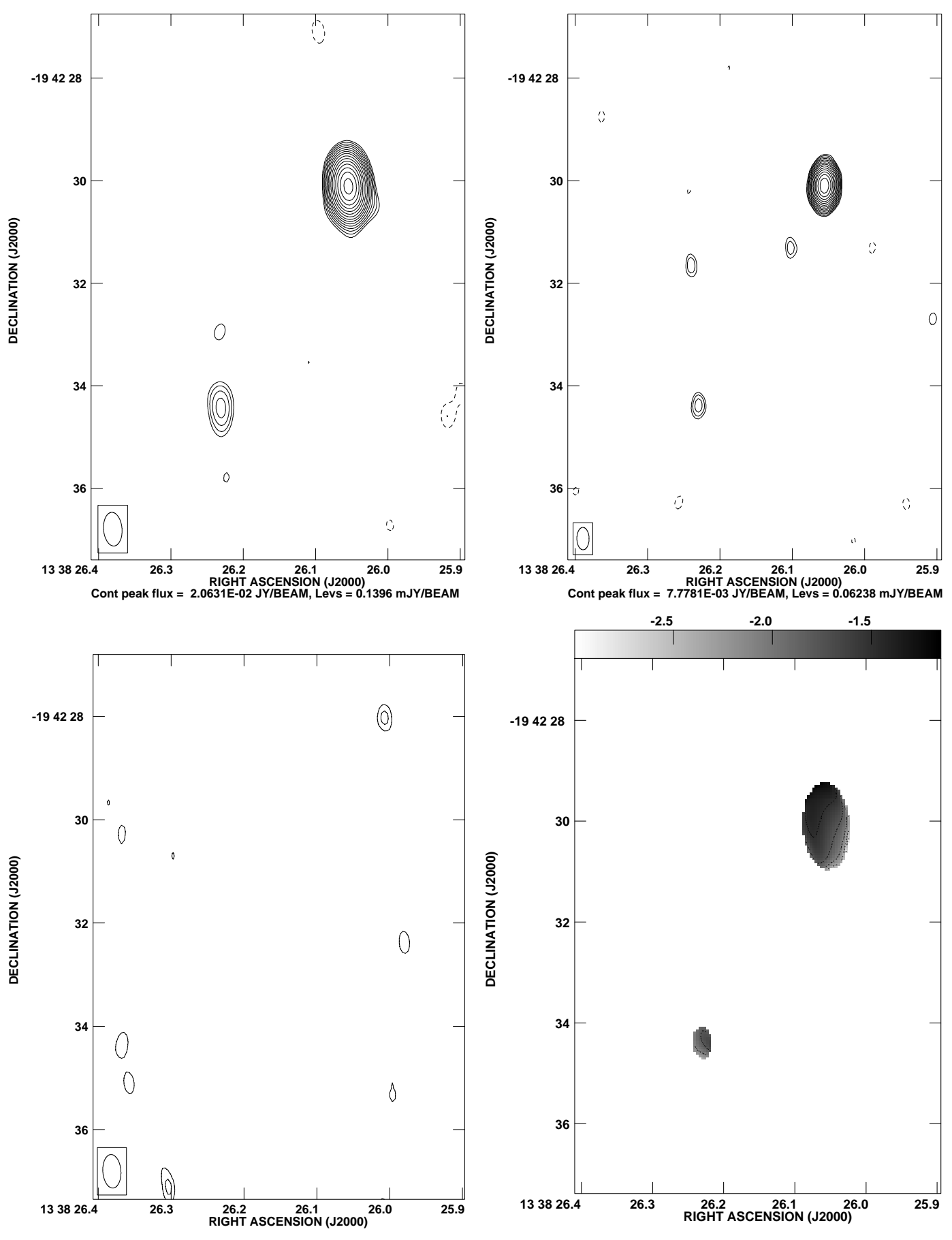

Fig. 18. Maps of the radio source 1338-19 at redshift $z=4.11$. The sequence of figures is the same as in Fig. 6 . The first contour level and the peak surface brightness are respectively $0.140 \mathrm{mJy}^{\text {beam }}{ }^{-1}$ and $21 \mathrm{mJy}^{\mathrm{beam}}{ }^{-1}$ for the $4.5 \mathrm{GHz} \mathrm{map} ; 0.074 \mathrm{mJy}$ beam $^{-1}$ and $8 \mathrm{mJy}_{\text {beam }}{ }^{-1}$ for the $8.2 \mathrm{GHz}$ map; $0.072 \mathrm{mJy}_{\text {beam }}{ }^{-1}$ for the $4.7 \mathrm{GHz}$ polarized intensity map 

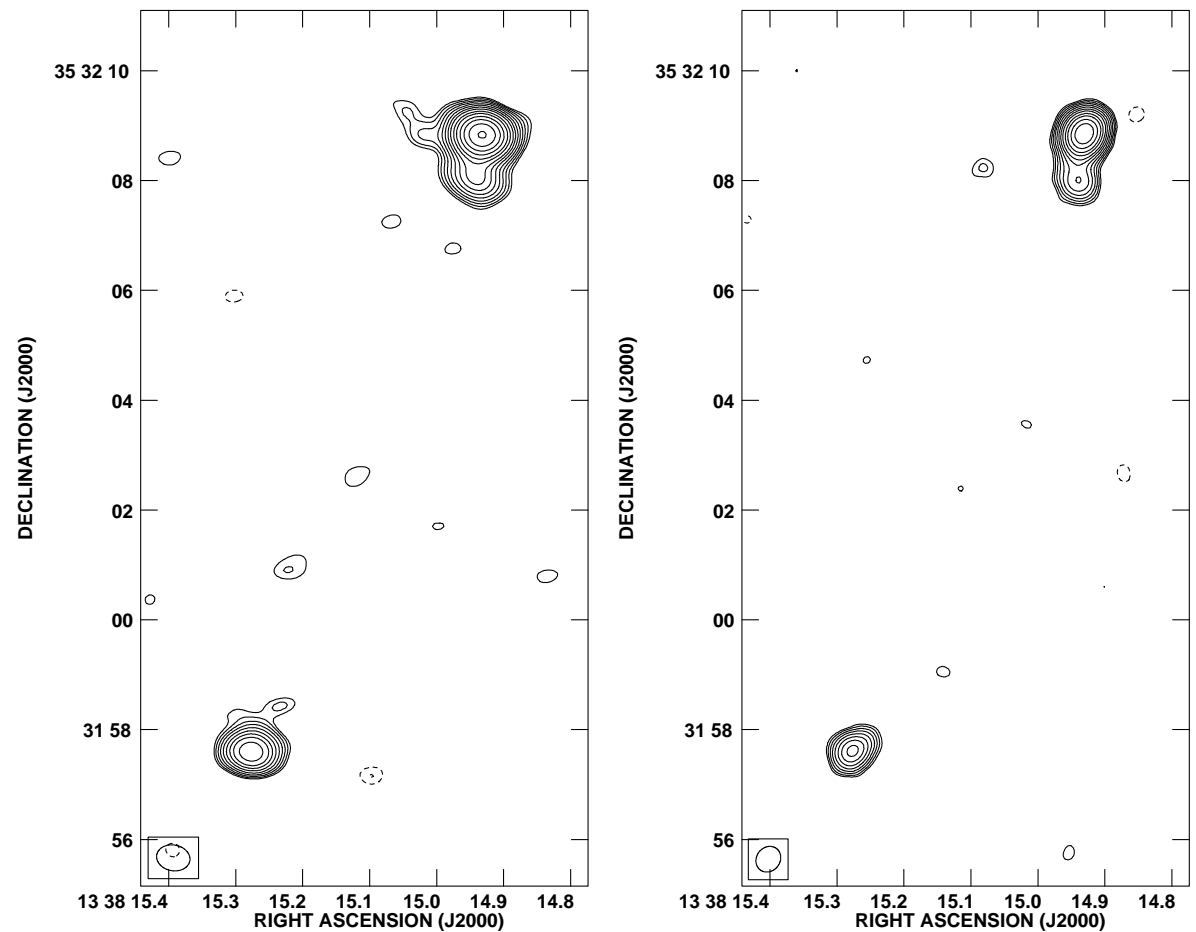

Cont peak flux $=1.2421 \mathrm{E}-02 \mathrm{JY} / \mathrm{BEAM}$, Levs $=0.1317 \mathrm{mJY} / \mathrm{BEAM}$ Cont peak flux $=5.2891 \mathrm{E}-03 \mathrm{JY} / \mathrm{BEAM}$, Levs $=0.08446 \mathrm{mJY} / \mathrm{BEAM}$
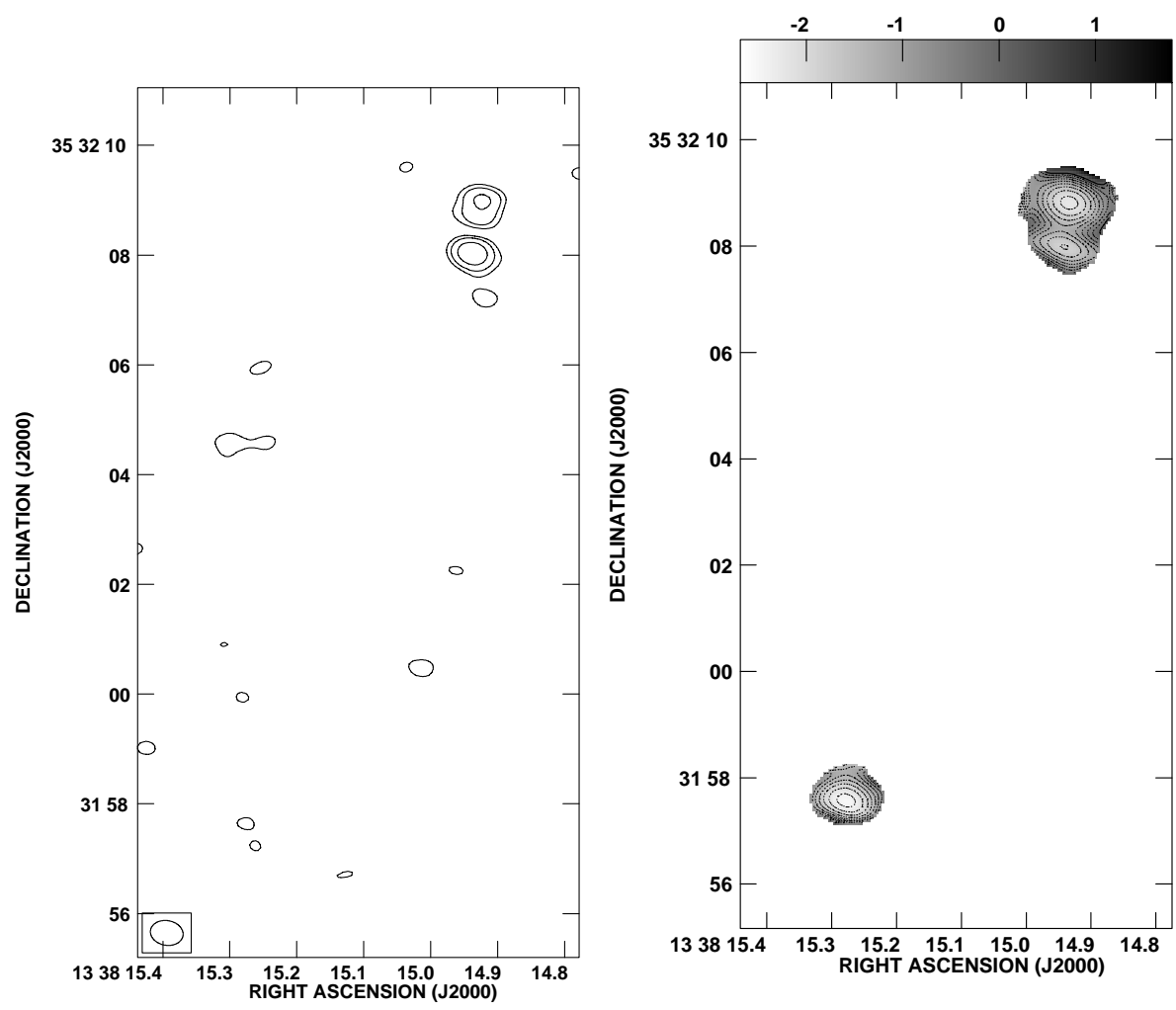

Peak flux $=5.5983 \mathrm{E}-04 \mathrm{JY} / \mathrm{BEAM}$, Levs $=0.07781 \mathrm{mJY} / \mathrm{BEAM}$

Fig. 19. Maps of the radio source $1339+35$ at redshift $z=2.772$. The sequence of figures is the same as in Fig. 6 . The first contour level and the peak surface brightness are respectively $0.132 \mathrm{mJy}_{\text {beam }}^{-1}$ and $12 \mathrm{mJy}$ beam $^{-1}$ for the $4.5 \mathrm{GHz}$ map; $0.084 \mathrm{mJy}$ beam $^{-1}$ and 5.3 mJy beam $^{-1}$ for the $8.2 \mathrm{GHz}$ map; $0.078 \mathrm{mJy}_{\text {beam }}^{-1}$ and 0.6 mJy beam $^{-1}$ for the $4.7 \mathrm{GHz}$ polarized intensity map 

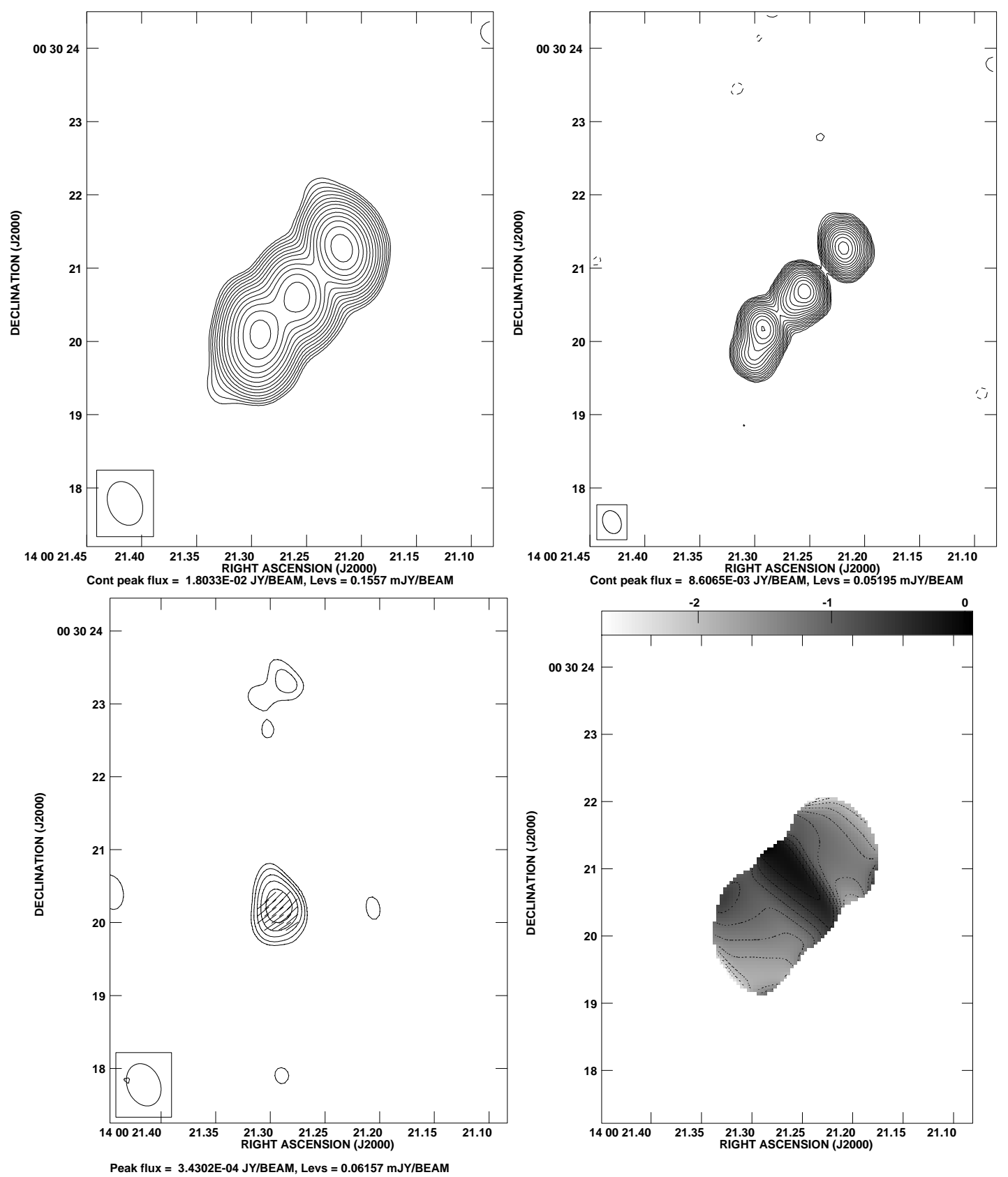

Fig. 20. Maps of the radio source $1357+007$ at redshift $z=2.671$. The sequence of figures is the same as in Fig. 6 . The first contour level and the peak surface brightness are respectively $0.156 \mathrm{mJy}^{\mathrm{beam}}{ }^{-1}$ and $18 \mathrm{mJy} \mathrm{beam}^{-1}$ for the $4.5 \mathrm{GHz}$ map; $0.052 \mathrm{mJy}_{\text {beam }}{ }^{-1}$ and $8.6 \mathrm{mJy}_{\text {beam }}{ }^{-1}$ for the $8.2 \mathrm{GHz}$ map; $0.062 \mathrm{mJy}_{\text {beam }}{ }^{-1}$ and $0.3 \mathrm{mJy} \mathrm{beam}^{-1}$ for the $4.7 \mathrm{GHz}$ polarized intensity map 

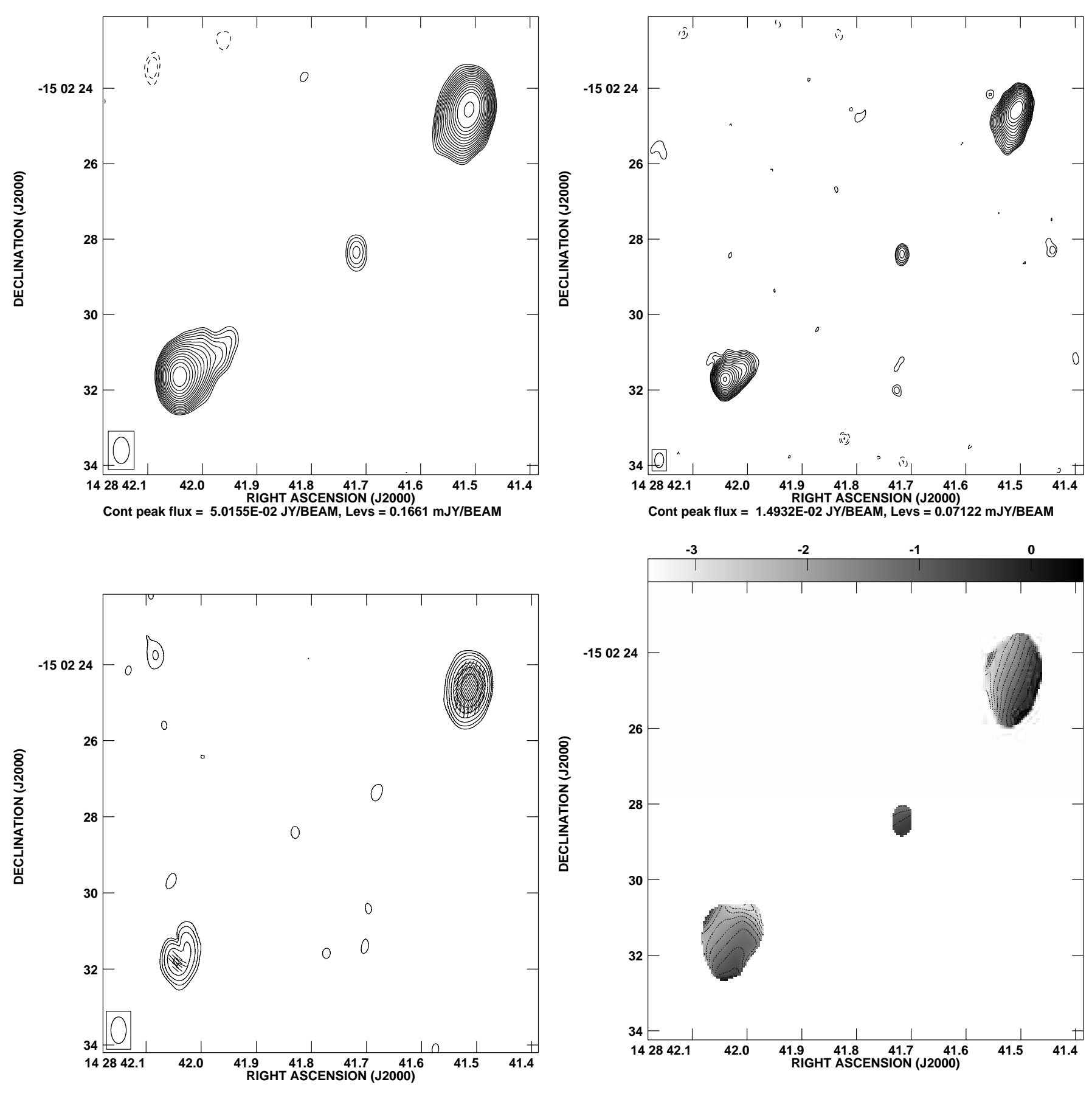

Peak flux $=8.7534 \mathrm{E}-03 \mathrm{JY} / \mathrm{BEAM}$, Levs $=0.07766 \mathrm{mJY} / \mathrm{BEAM}$

Fig. 21. Maps of the radio source $1425-148$ at redshift $z=2.355$. The sequence of figures is the same as in Fig. 6. The first contour level and the peak surface brightness are respectively $0.166 \mathrm{mJy}^{\mathrm{beam}}{ }^{-1}$ and $50 \mathrm{mJy} \mathrm{beam}^{-1}$ for the $4.5 \mathrm{GHz}$ map;

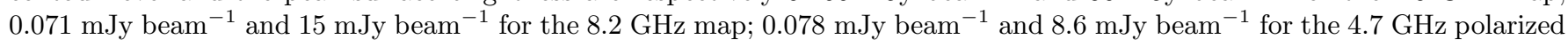
intensity map 

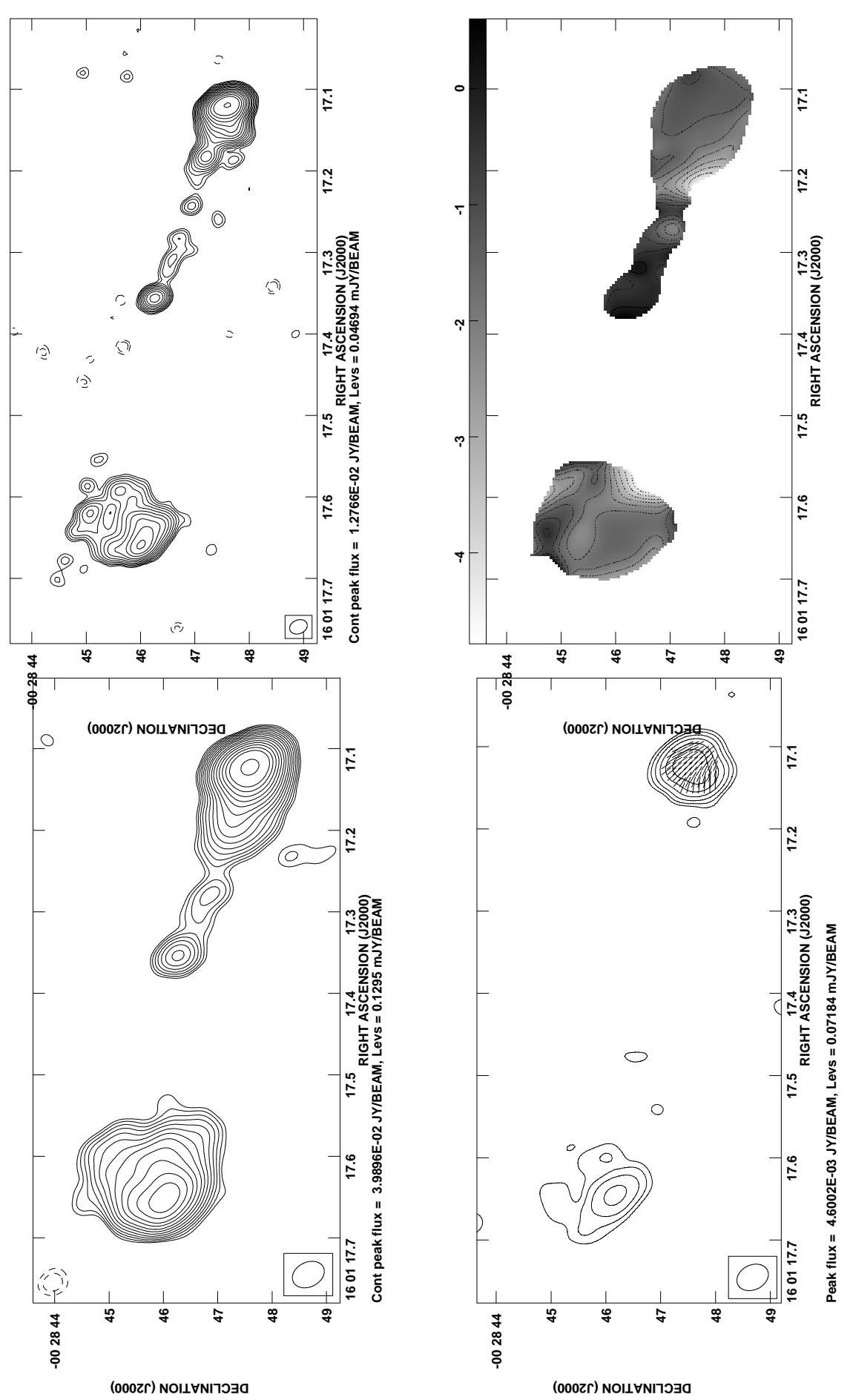

Fig. 22. Maps of the radio source $1558-003$ at redshift $z=2.52$. The sequence of figures is the same as in Fig. 6 . The first contour level and the peak surface brightness are respectively $0.129 \mathrm{mJy}^{\text {beam }}{ }^{-1}$ and $40 \mathrm{mJy}$ beam $^{-1}$ for the $4.5 \mathrm{GHz}$ map;

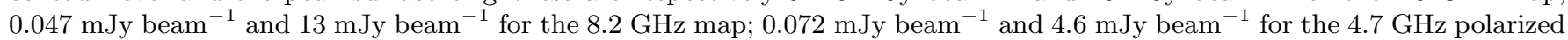
intensity map 

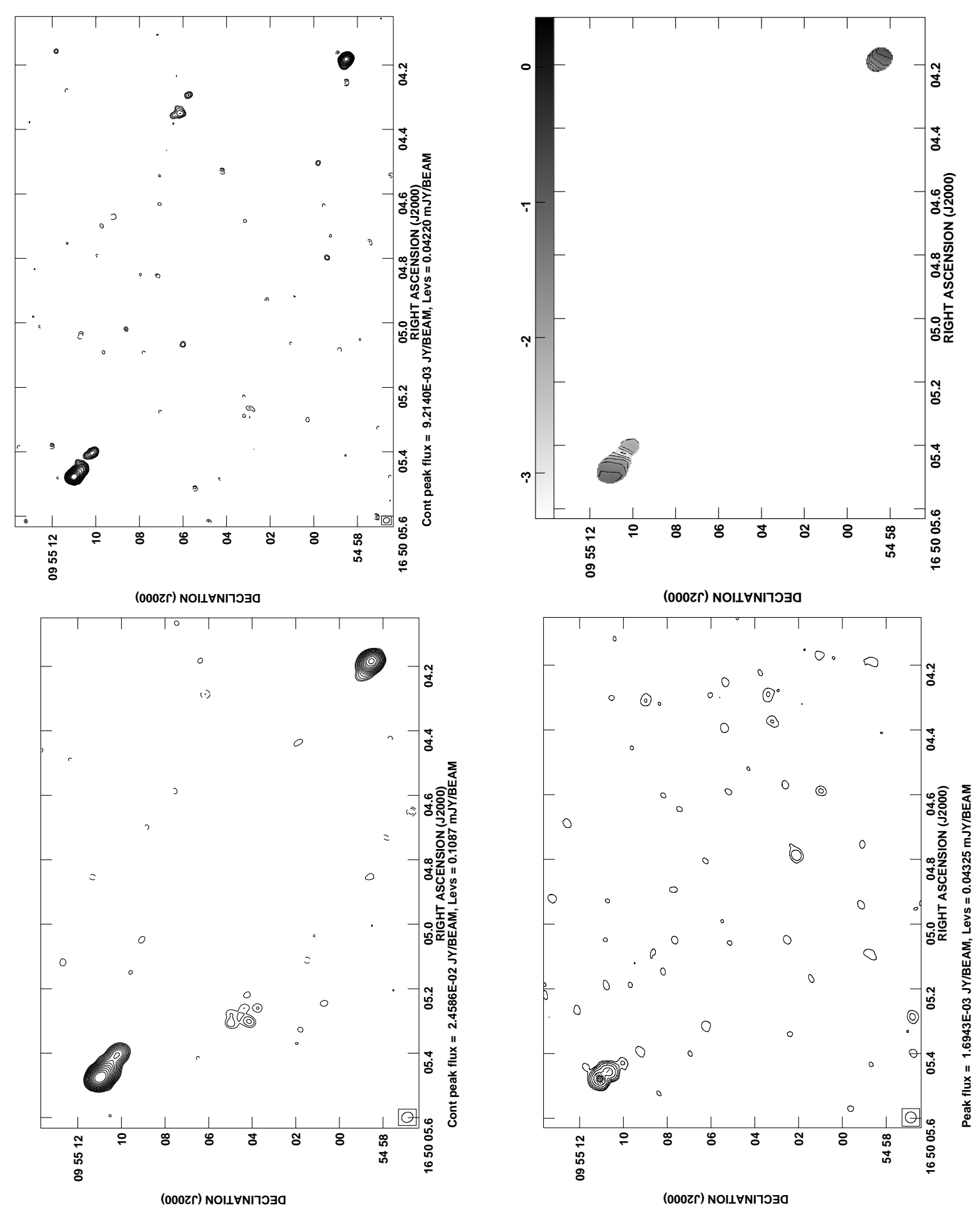

Fig. 23. Maps of the radio source $1647+100$ at redshift $z=2.509$. The sequence of figures is the same as in Fig. 6. The first contour level and the peak surface brightness are respectively $0.109 \mathrm{mJy}^{\text {beam }}{ }^{-1}$ and 24 . mJy beam ${ }^{-1}$ for the $4.5 \mathrm{GHz}^{\mathrm{map}}$;

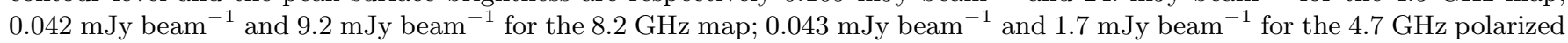
intensity map 

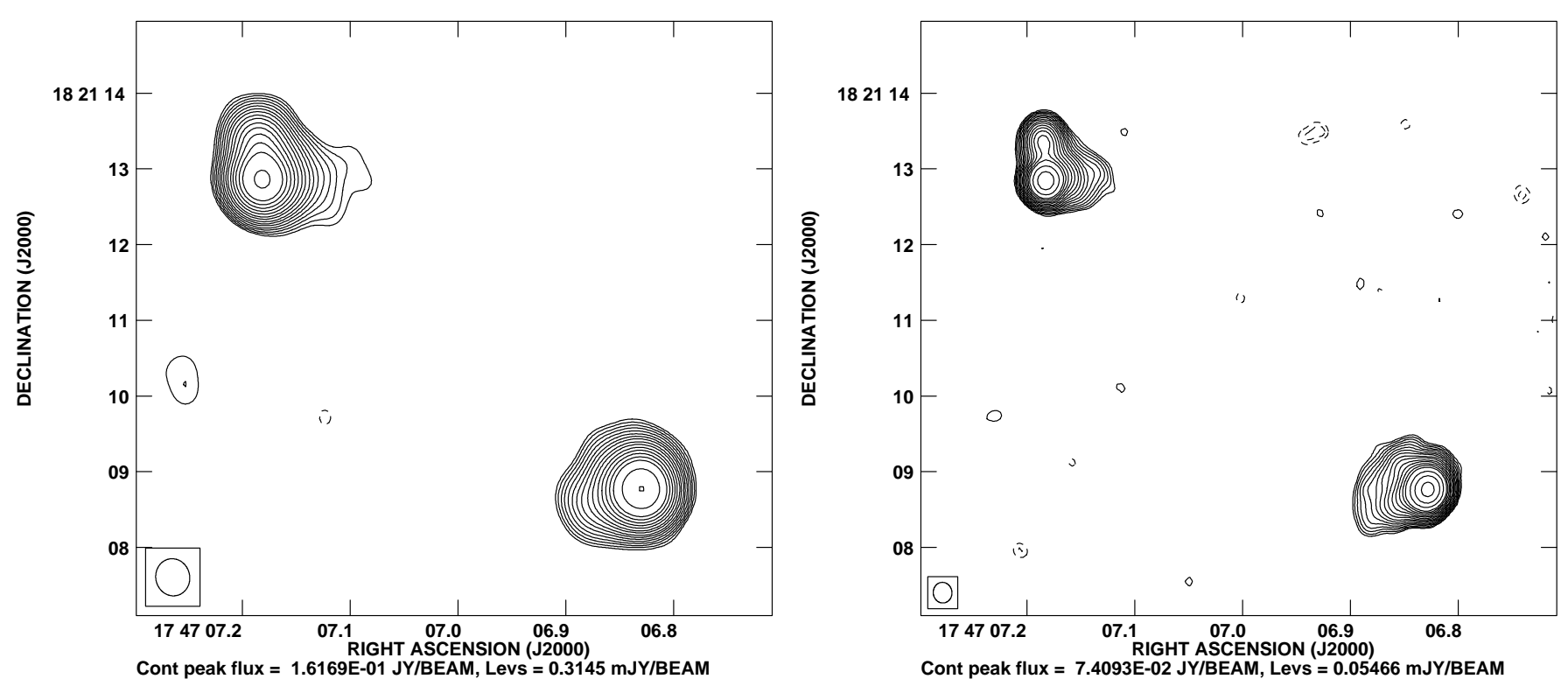

Cont peak flux $=7.4093 \mathrm{E}-02$ JY/BEAM, Levs $=0.05466 \mathrm{mJY} / \mathrm{BEAM}$
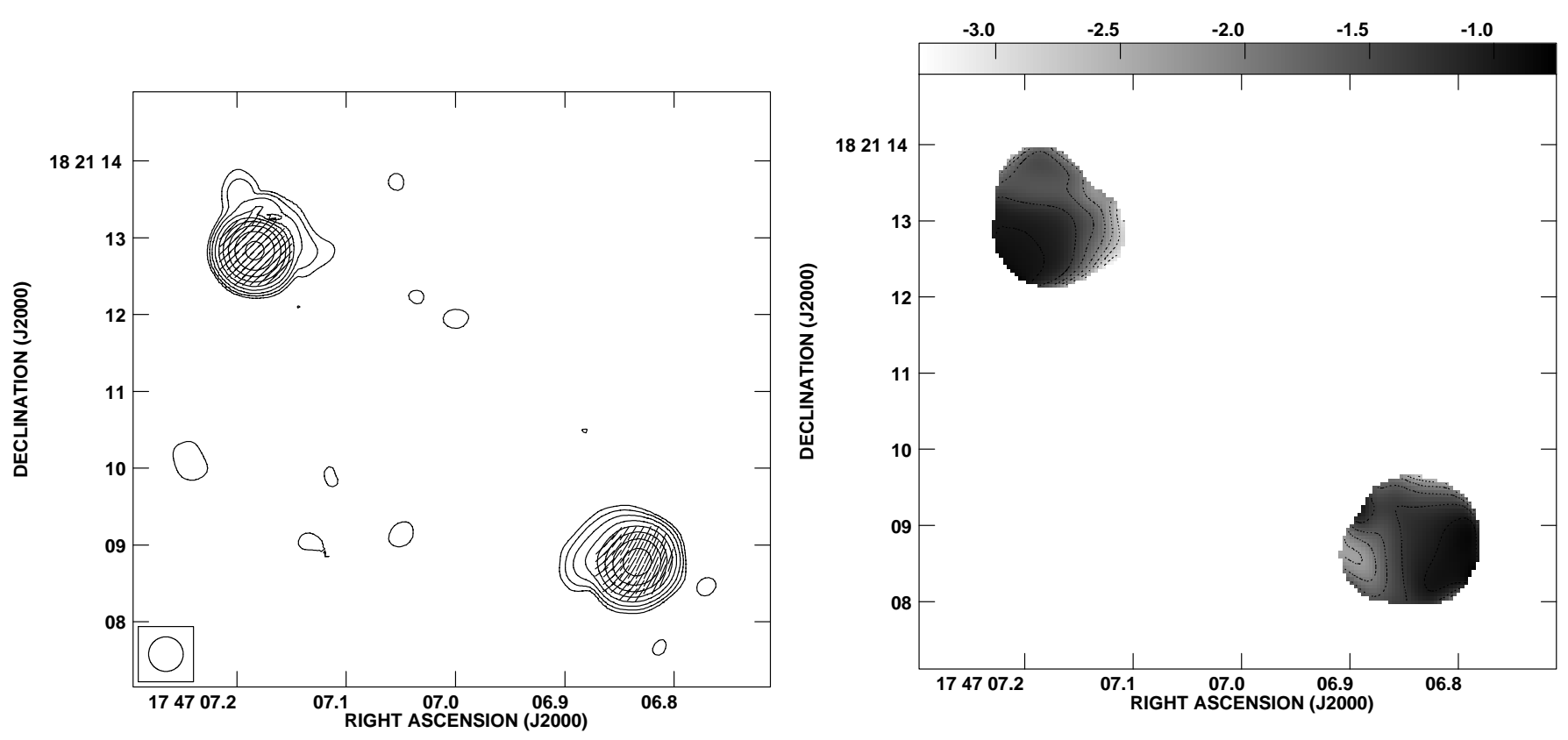

Peak flux $=2.0396 \mathrm{E}-02$ JY/BEAM, Levs $=0.06523 \mathrm{mJY} / \mathrm{BEAM}$

Fig. 24. Maps of the radio source $1747+182$ at redshift $z=2.281$. The sequence of figures is the same as in Fig. 6 . The first contour level and the peak surface brightness are respectively $0.314 \mathrm{mJy}_{\text {beam }}{ }^{-1}$ and $162 \mathrm{mJy}$ beam ${ }^{-1}$ for the $4.5 \mathrm{GHz} \mathrm{map}$;

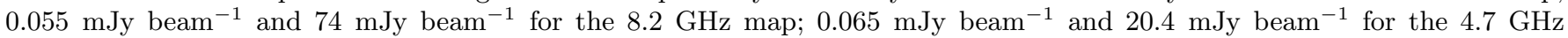
polarized intensity map 

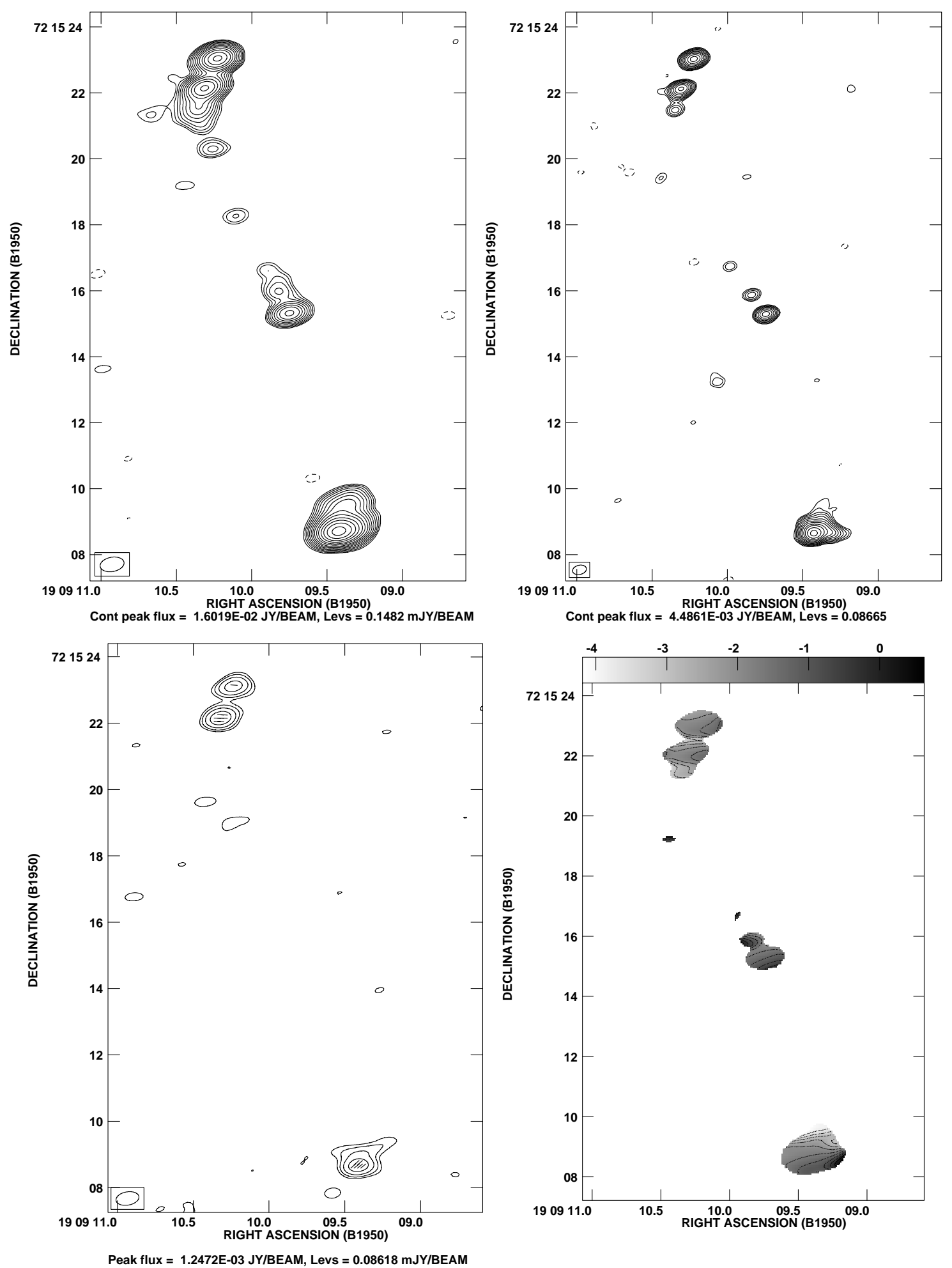

Fig. 25. Maps of the radio source $1908+722$ at redshift $z=3.537$. The sequence of figures is the same as in Fig. 6. The first contour level and the peak surface brightness are respectively $0.148 \mathrm{mJy} \mathrm{beam}^{-1}$ and $16 \mathrm{mJy}$ beam ${ }^{-1}$ for the $4.5 \mathrm{GHz}$ map; $0.087 \mathrm{mJy}_{\text {beam }^{-1}}$ and $4.5 \mathrm{mJy}_{\text {beam }}{ }^{-1}$ for the $8.2 \mathrm{GHz}$ map; $0.086 \mathrm{mJy}_{\text {beam }}{ }^{-1}$ and $1.2 \mathrm{mJy} \mathrm{beam}^{-1}$ for the $4.7 \mathrm{GHz}$ polarized intensity map 

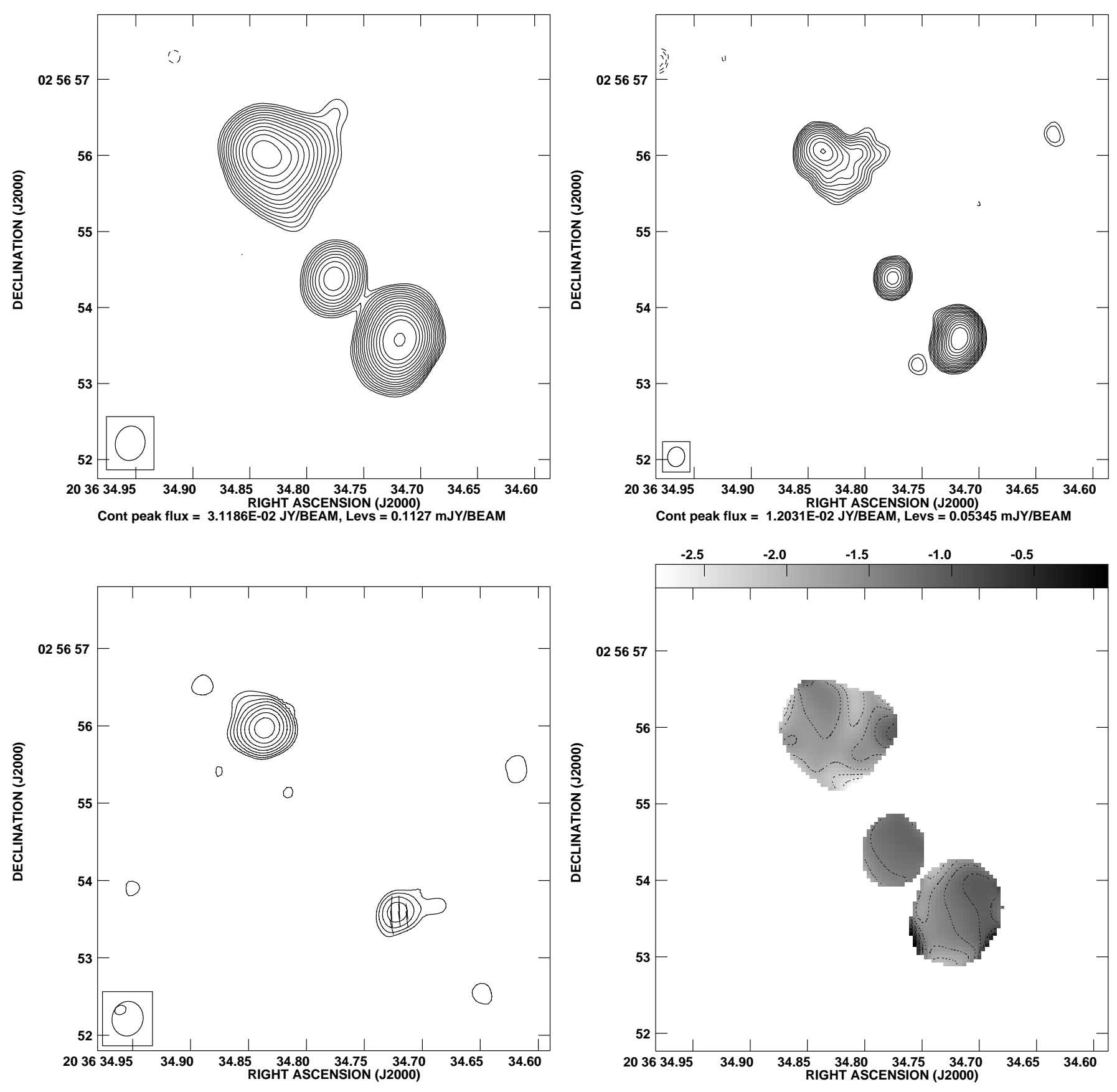

Fig. 26. Maps of the radio source $2034+027$ at redshift $z=2.129$. The sequence of figures is the same as in Fig. 6 . The first contour level and the peak surface brightness are respectively $0.113 \mathrm{mJy}_{\text {beam }}{ }^{-1}$ and $31 \mathrm{mJy}$ beam $^{-1}$ for the $4.5 \mathrm{GHz}$ map;

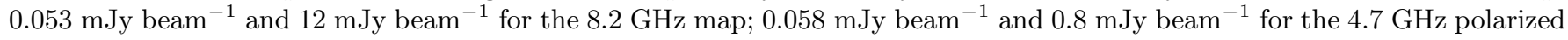
intensity map 

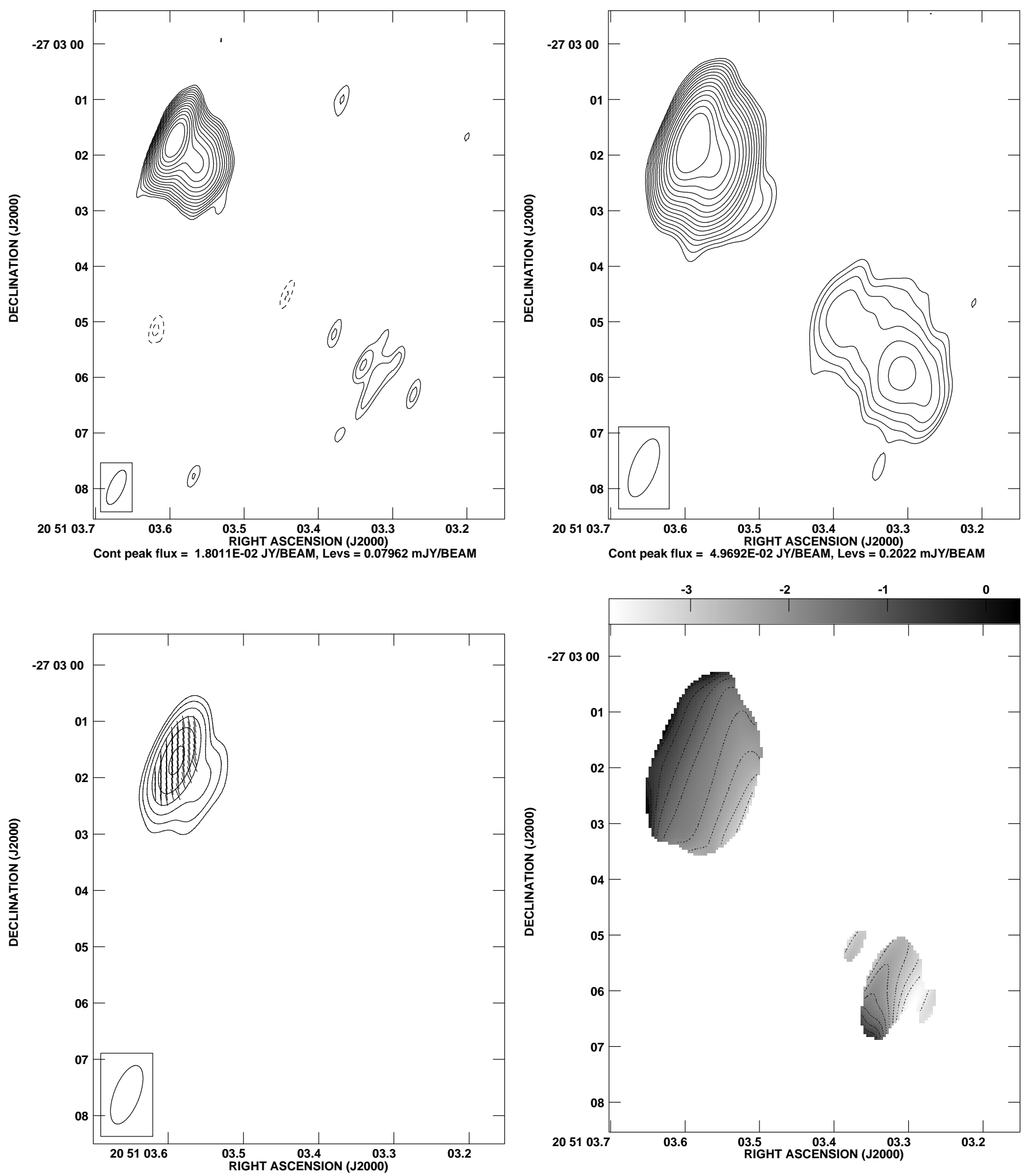

Peak flux $=4.0599 \mathrm{E}-03 \mathrm{JY} / \mathrm{BEAM}$, Levs $=0.1081 \mathrm{mJY} / \mathrm{BEAM}$

Fig. 27. Maps of the radio source 2048-272 at redshift $z=2.06$. The sequence of figures is the same as in Fig. 6. The first contour level and the peak surface brightness are respectively $0.200 \mathrm{mJy}^{\mathrm{beam}}{ }^{-1}$ and $50 \mathrm{mJy} \mathrm{beam}^{-1}$ for the $4.5 \mathrm{GHz}$ map; $0.079 \mathrm{mJy} \mathrm{beam}^{-1}$ and $18 \mathrm{mJy}_{\text {beam }}{ }^{-1}$ for the $8.2 \mathrm{GHz}$ map; $0.108 \mathrm{mJy}_{\text {beam }}{ }^{-1}$ and $4.1 \mathrm{mJy}$ beam $^{-1}$ for the $4.7 \mathrm{GHz}$ polarized intensity map 

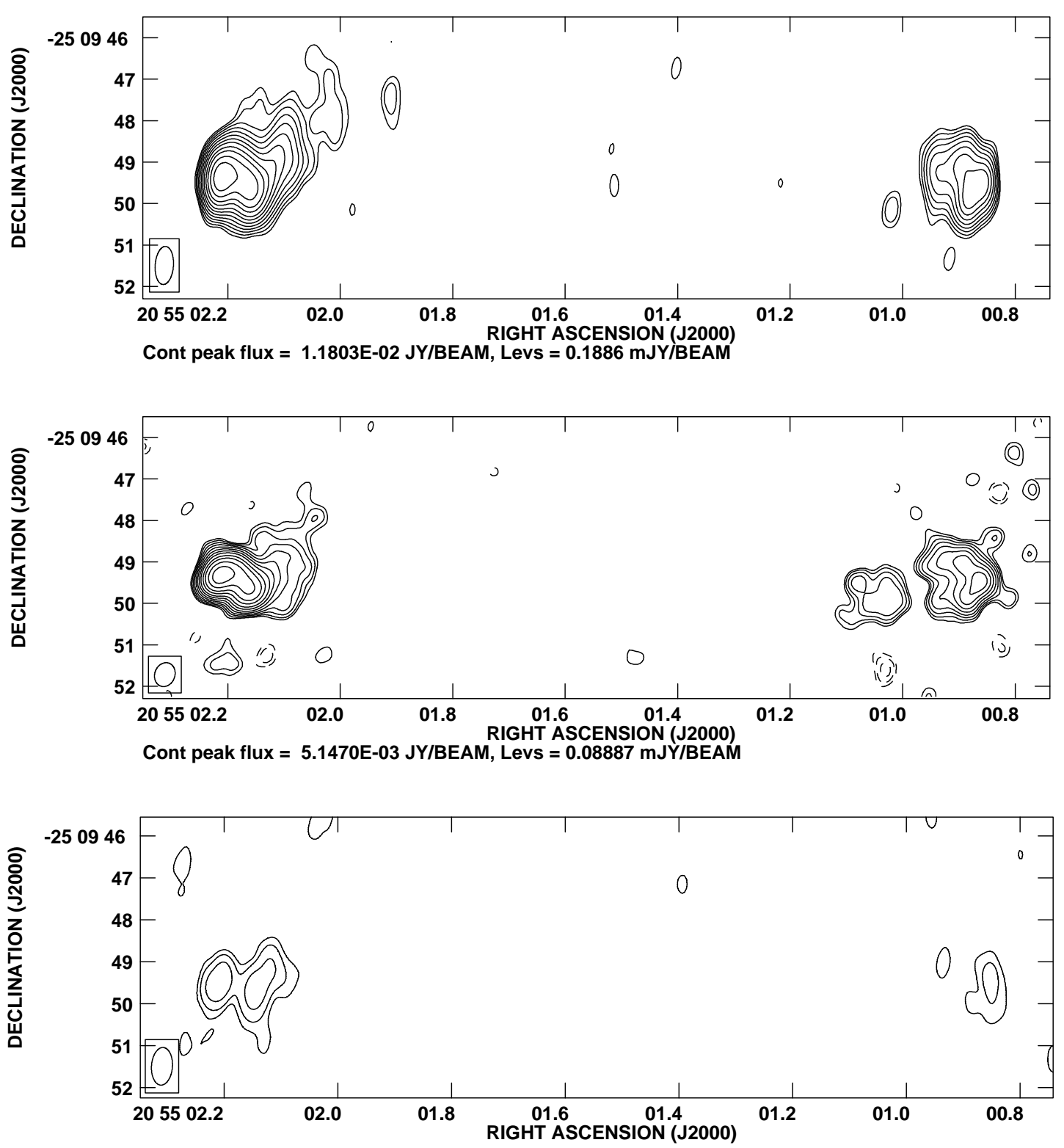

Peak flux $=7.9026 \mathrm{E}-04$ JY/BEAM, Levs $=0.1018 \mathrm{mJY} / \mathrm{BEAM}$

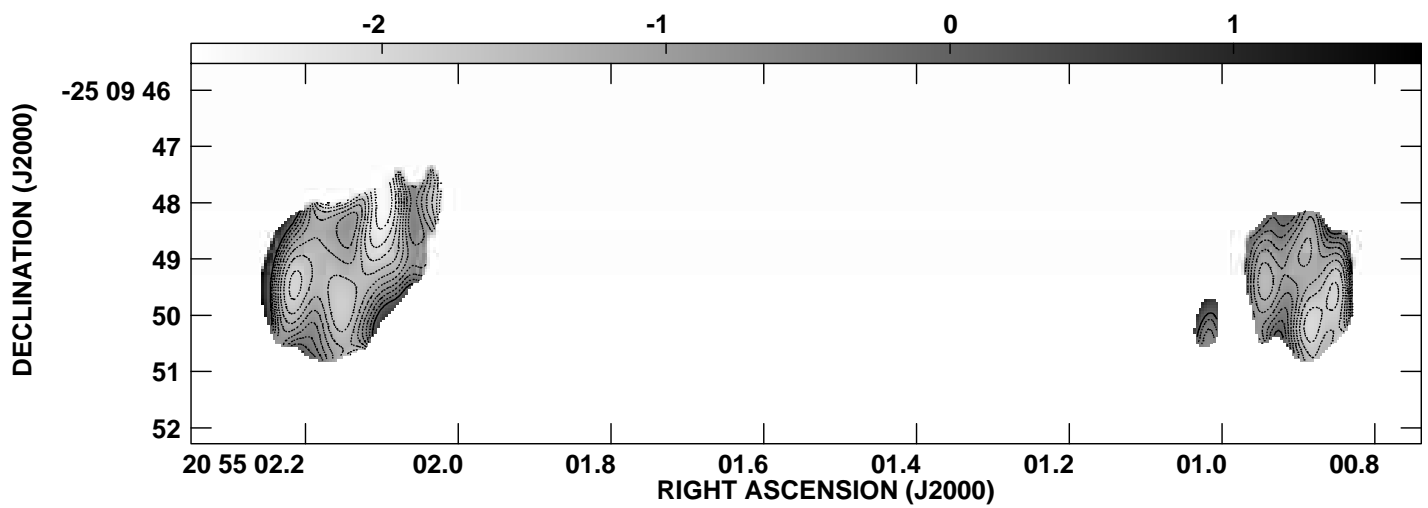

Fig. 28. Maps of the radio source 2052-253 at redshift $z=2.63$. The sequence of figures is the same as in Fig. 6. The first contour level and the peak surface brightness are respectively $0.188 \mathrm{mJy}^{\mathrm{beam}}{ }^{-1}$ and $11.8 \mathrm{mJy}^{\mathrm{beam}}{ }^{-1}$ for the $4.5 \mathrm{GHz}$ map; $0.088 \mathrm{mJy}_{\text {beam }^{-1}}$ and $5.1 \mathrm{mJy}$ beam $^{-1}$ for the $8.2 \mathrm{GHz}$ map; $0.102 \mathrm{mJy}_{\text {beam }}{ }^{-1}$ and $0.8 \mathrm{mJy} \mathrm{beam}^{-1}$ for the $4.7 \mathrm{GHz}$ polarized intensity map 

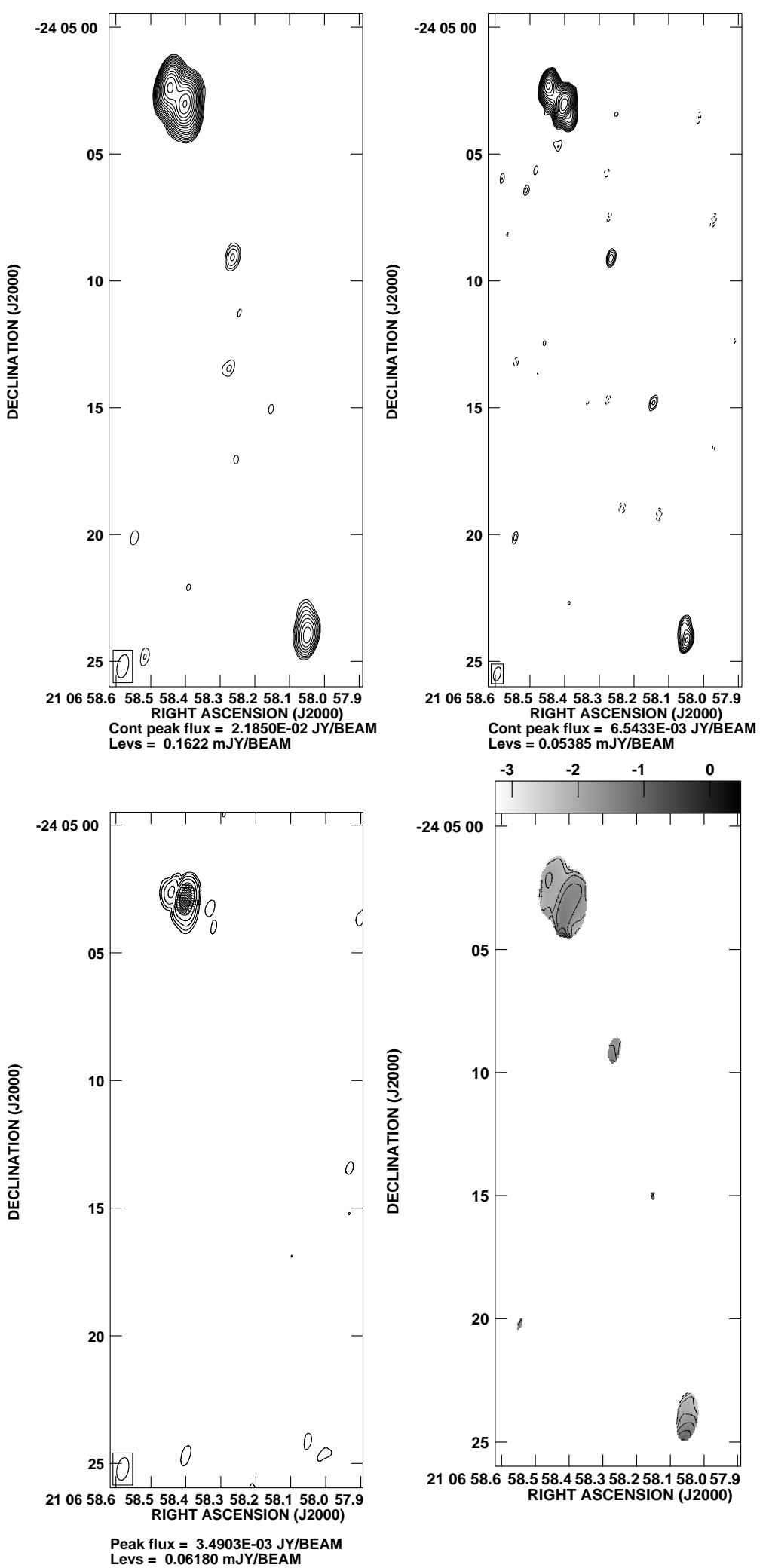

Fig. 29. Maps of the radio source 2104-242 at redshift $z=2.49$. The sequence of figures is the same as in Fig. 6. The first contour level and the peak surface brightness are respectively $0.162 \mathrm{mJy}^{\mathrm{beam}}{ }^{-1}$ and $21.8 \mathrm{mJy} \mathrm{beam}^{-1}$ for the $4.5 \mathrm{GHz}$ map; $0.054 \mathrm{mJy}_{\text {beam }}{ }^{-1}$ and $6.5 \mathrm{mJy}_{\text {beam }}{ }^{-1}$ for the $8.2 \mathrm{GHz}$ map; $0.062 \mathrm{mJy}_{\text {beam }}{ }^{-1}$ and $3.5 \mathrm{mJy}$ beam ${ }^{-1}$ for the $4.7 \mathrm{GHz}$ polarized intensity map 

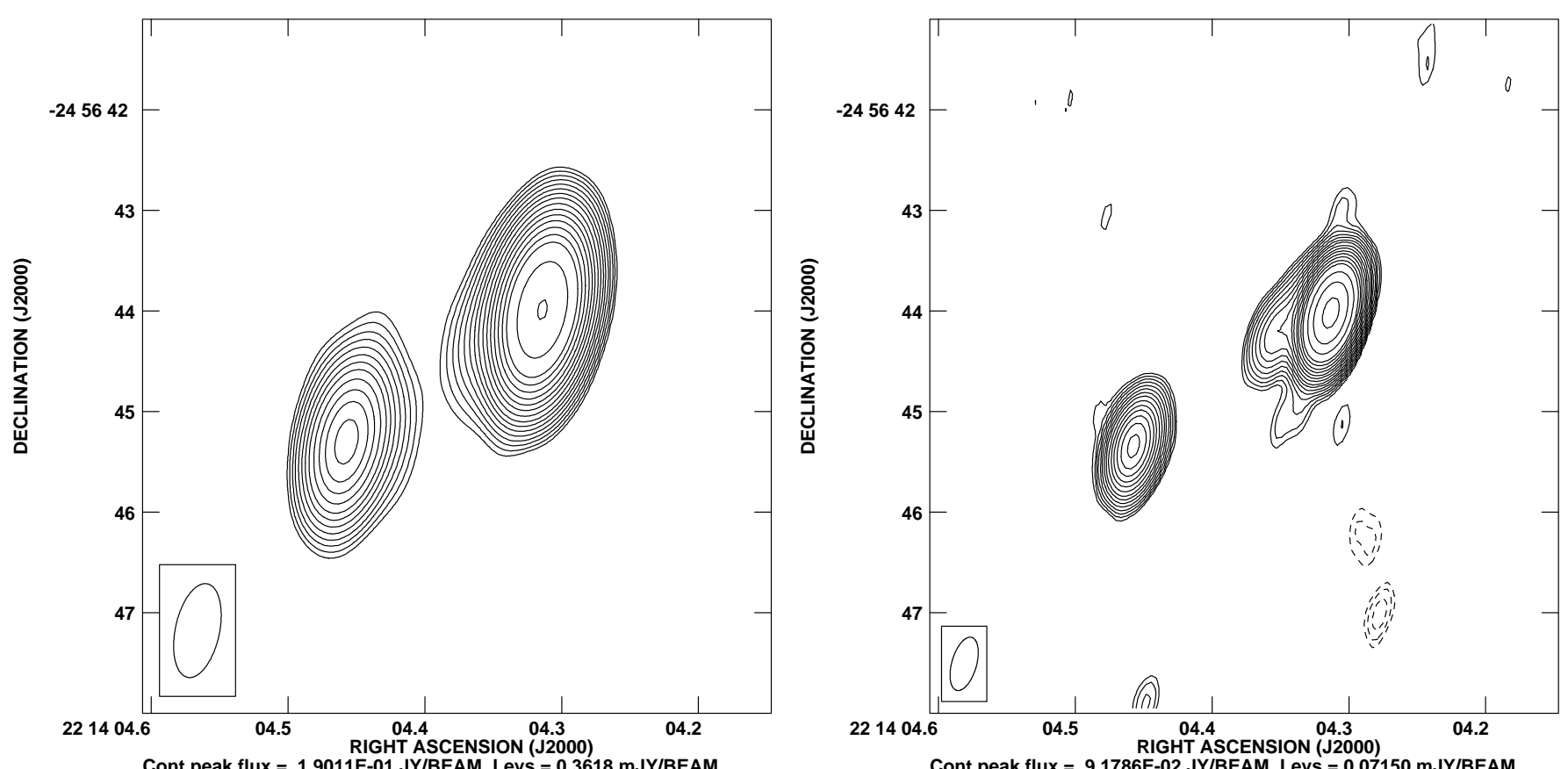

Cont peak flux $=1.9011 \mathrm{E}-01 \mathrm{JY} / \mathrm{BEAM}$, Levs $=0.3618 \mathrm{mJY} / \mathrm{BEAM}$

Cont peak flux $=9.1786 \mathrm{E}-02$ JY/BEAM, Levs $=0.07150 \mathrm{mJY} / \mathrm{BEAM}$
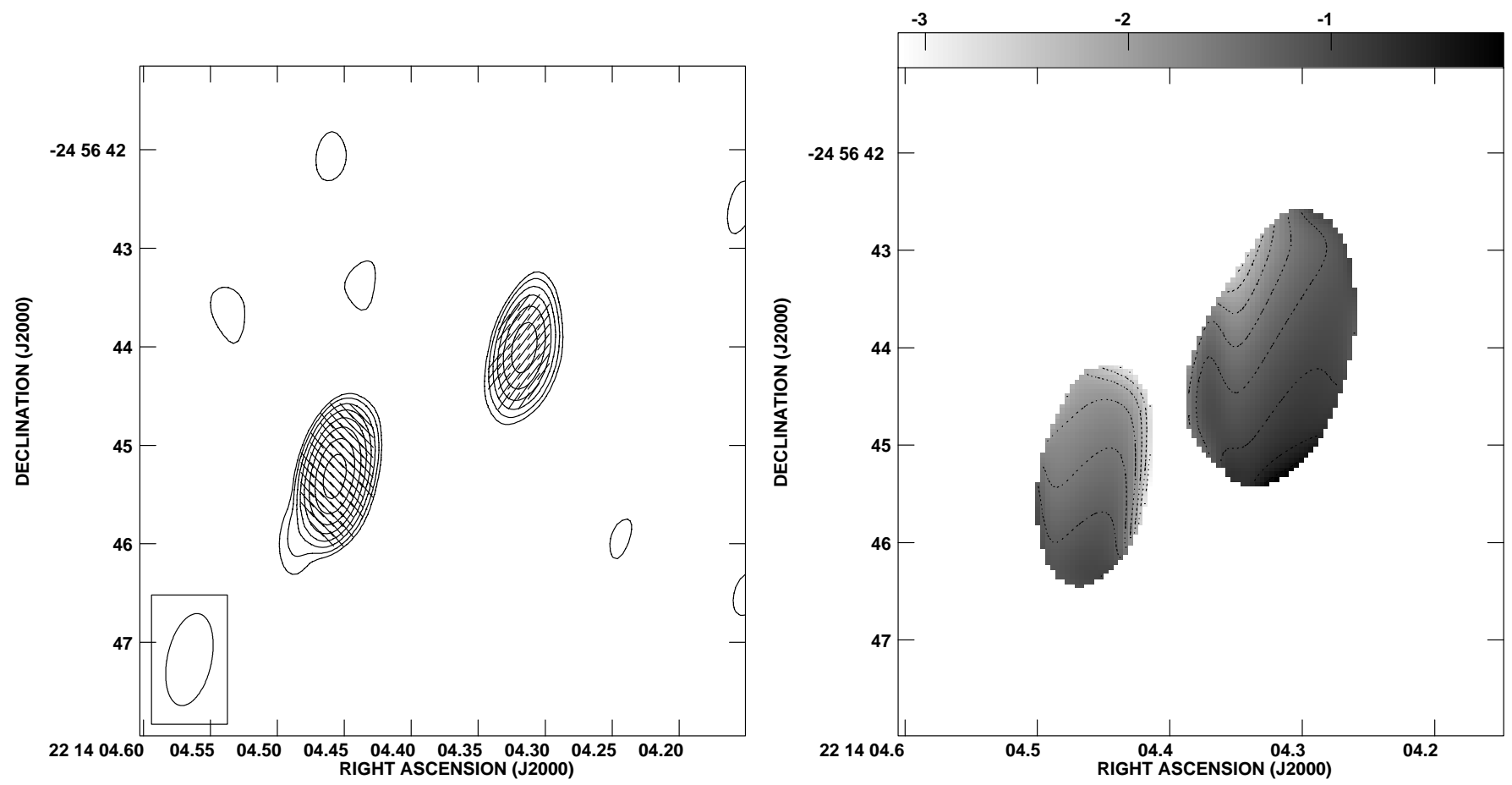

Peak flux $=1.1488 \mathrm{E}-03 \mathrm{JY} / \mathrm{BEAM}$, Levs $=0.08879 \mathrm{mJY} / \mathrm{BEAM}$

Fig. 30. Maps of the radio source $2211-251$ at redshift $z=2.508$. The sequence of figures is the same as in Fig. 6. The first contour level and the peak surface brightness are respectively $0.362 \mathrm{mJy}^{\text {beam }}{ }^{-1}$ and $190 \mathrm{mJy} \mathrm{beam}^{-1}$ for the $4.5 \mathrm{GHz}$ map; $0.071 \mathrm{mJy}$ beam $^{-1}$ and $92 \mathrm{mJy}$ beam $^{-1}$ for the $8.2 \mathrm{GHz}$ map; $0.089 \mathrm{mJy}^{\text {beam }}{ }^{-1}$ and $1.1 \mathrm{mJy} \mathrm{beam}^{-1}$ for the $4.7 \mathrm{GHz}$ polarized intensity map 

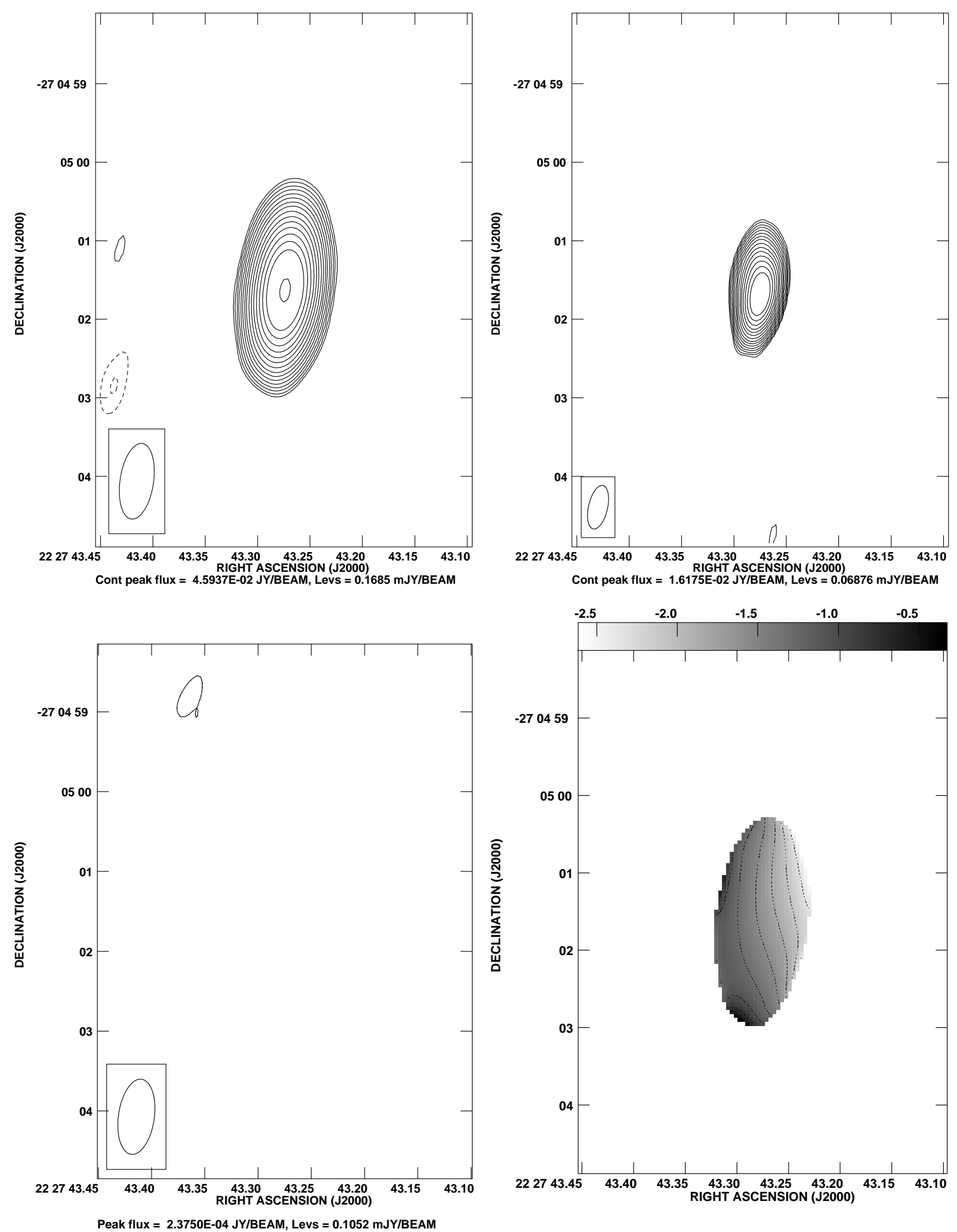

Fig. 31. Maps of the radio source $2224-273$ at redshift $z=1.68$. The sequence of figures is the same as in Fig. 6. The first contour level and the peak surface brightness are respectively $0.168 \mathrm{mJy}^{\mathrm{beam}}{ }^{-1}$ and $162 \mathrm{mJy} \mathrm{beam}^{-1}$ for the $4.5 \mathrm{GHz}$ map; $0.068 \mathrm{mJy}$ beam $^{-1}$ and $45.9 \mathrm{mJy}^{\text {beam }}{ }^{-1}$ for the $8.2 \mathrm{GHz}$ map; $0.105 \mathrm{mJy}^{\text {beam }}{ }^{-1}$ for the $4.7 \mathrm{GHz}$ polarized intensity map 

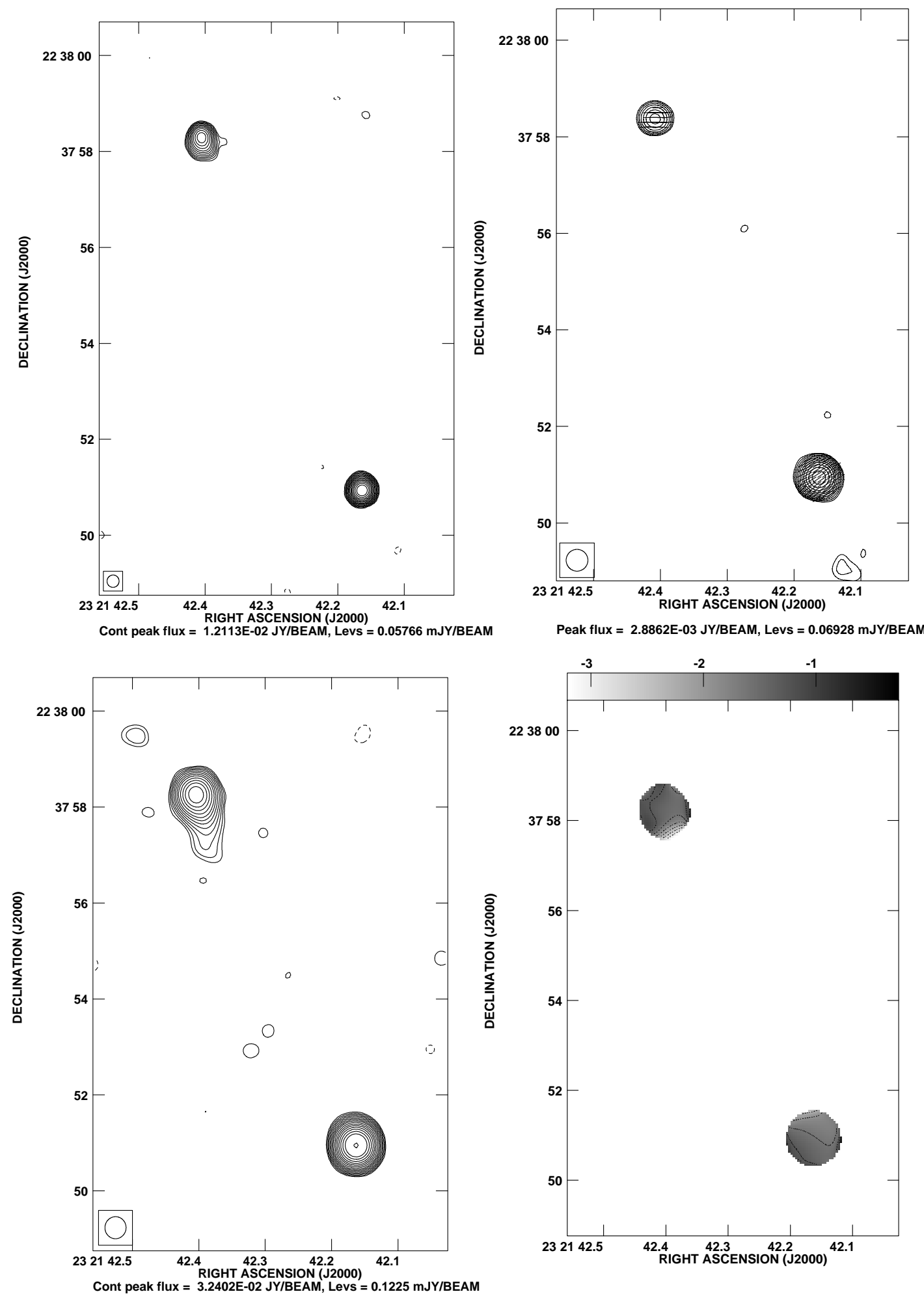

Fig. 32. Maps of the radio source $2319+223$ at redshift $z=2.554$. The sequence of figures is the same as in Fig. 6. The first contour level and the peak surface brightness are respectively $0.122 \mathrm{mJy}_{\text {beam }}{ }^{-1}$ and $32 \mathrm{mJy}$ beam $^{-1}$ for the $4.5 \mathrm{GHz}$ map;

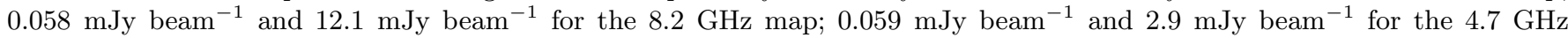
polarized intensity map 